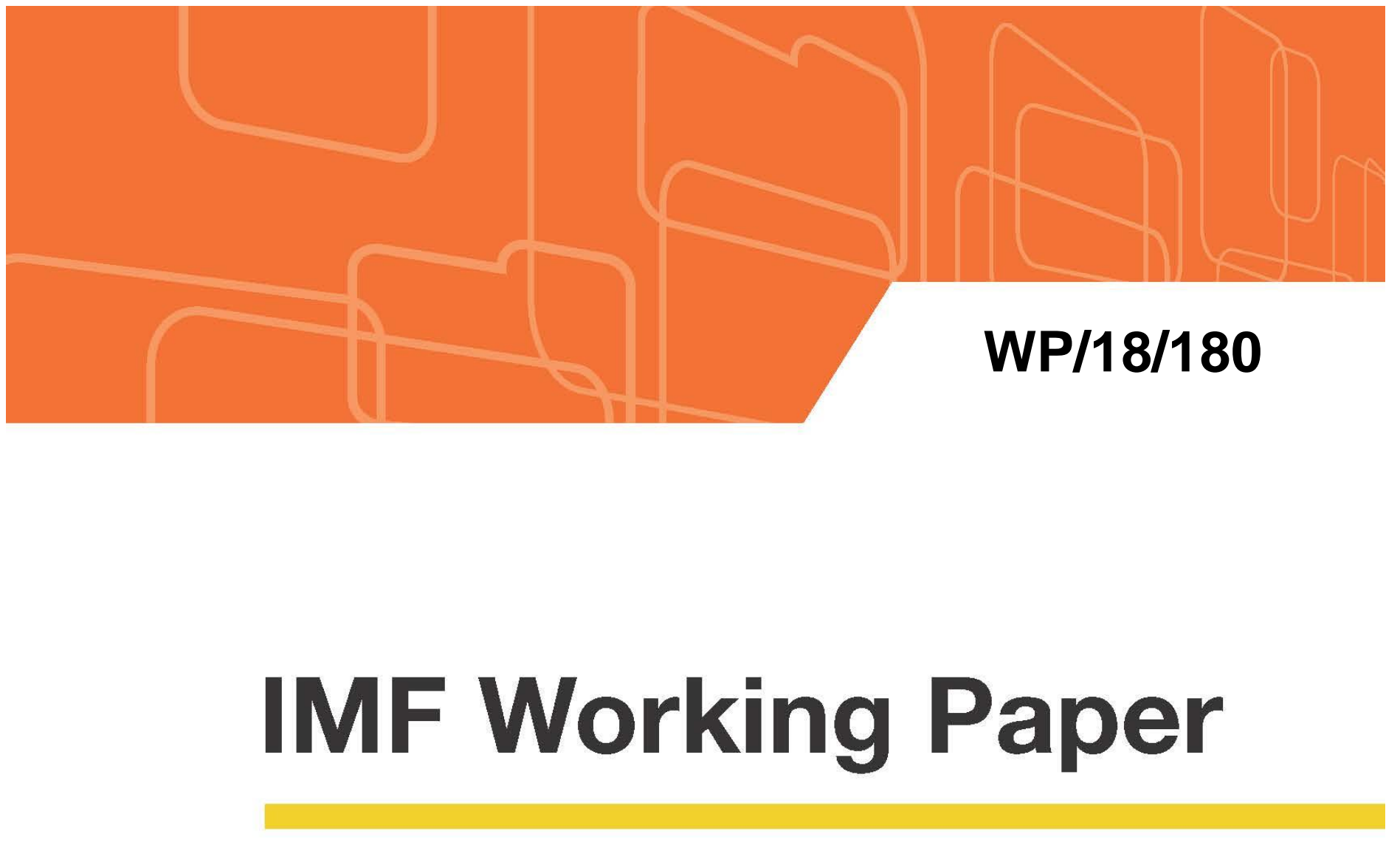

\title{
The Term Structure of Growth-at-Risk
}

by Tobias Adrian, Federico Grinberg, Nellie Liang, Sheheryar Malik, Jie Yu

IMF Working Papers describe research in progress by the author(s) and are published to elicit comments and to encourage debate. The views expressed in IMF Working Papers are those of the author(s) and do not necessarily represent the views of the IMF, its Executive Board, or IMF management. 


\title{
IMF Working Paper
}

Monetary and Capital Markets Department

The Term Structure of Growth-at-Risk ${ }^{1}$

Prepared by Tobias Adrian, Federico Grinberg, Nellie Liang, Sheheryar Malik, Jie Yu

August 2018

\begin{abstract}
IMF Working Papers describe research in progress by the author(s) and are published to elicit comments and to encourage debate. The views expressed in IMF Working Papers are those of the author(s) and do not necessarily represent the views of the IMF, its Executive Board, or IMF management.
\end{abstract}

\begin{abstract}
We show that the conditional distribution of forecasted GDP growth depends on financial conditions in a panel of 11 advanced economies. Financial conditions have a larger effect on the lower 5th percentile of conditional growth — which we call growth-at-risk (GaR) - than the median. In addition, the term structure of $\mathrm{GaR}$ reflects that when initial financial conditions are loose, downside risks are lower in the near-term but increase in later quarters. This intertemporal tradeoff for loose financial conditions is amplified when credit-to-GDP growth is rapid. Using granular instrumental variables, we also provide evidence that the relationship from loose financial conditions to future downside risks is causal. Our results suggest that models of macrofinancial linkages should incorporate the endogeneity of higherorder moments to systematically account for downside risks to growth in the medium run.

JEL Classification Numbers: G1, E1, F44

Keywords: downside risk, macrofinancial linkages, volatility paradox, financial stability, quantile regression

Author's E-Mail Address: tadrian@imf.org, fgrinberg@imf.org, jnliang@brookings.edu, smalik2@imf.org, jie.eva.yu@gmail.com

\footnotetext{
${ }^{1}$ We thank Fernando Duarte, Tommaso Mancini Griffoli, Michael Kiley, Andreas Lehnert, Frank Schorfheide, Pawel Zabcyzk and participants at a BIS Research Network conference and an IMF workshop on GDP-at-risk, other IMF colleagues for helpful comments, an anonymous referee and editor.
} 
Contents

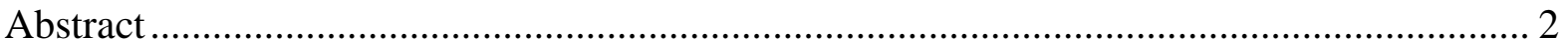

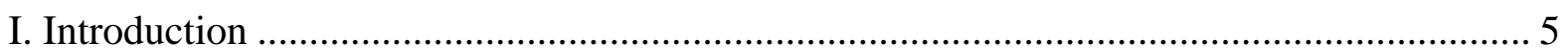

II. Modeling Growth-at-Risk .................................................................................... 10

A. Model Estimation with Quantile Regressions....................................................... 10

B. Financial Conditions and Credit-to-GDP Growth.................................................. 13

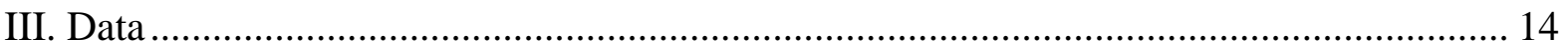

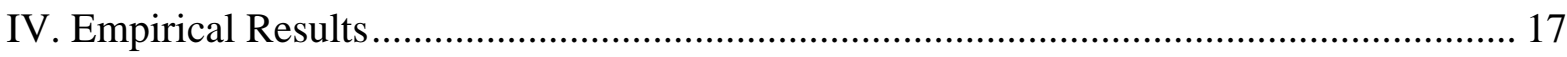

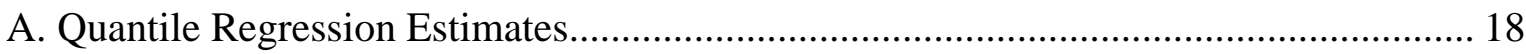

B. Time Series of Average GaR ........................................................................ 20

C. Probability Density Functions of Forecasted Growth and GaR ................................. 21

D. Term Structures of GaR by Initial FCI Groups..................................................... 25

E. Term Structures of Predicted Median Growth and GaR by Initial FCI Groups............. 27

F. Applying Granular Instrumental Variables to Evaluate Causality ............................... 30

G. Interpreting the Intertemporal Risk-Return Tradeoff.............................................. 33

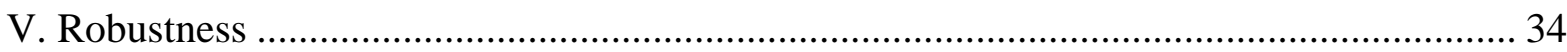

A. Alternative Financial Indicator and Credit Variables ............................................... 35

B. Quantile Estimates Excluding the Global Financial Crisis .......................................... 38

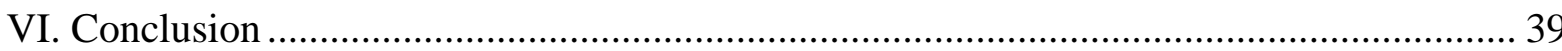

Figures

1. Estimated Coefficients on FCI, Credit Growth, and Credit Boom ...................................6

2. Predicted Average Conditional Distribution of Real GDP Growth ................................21

3. Probably Density Functions for Decile of Loosest FCI, by Projection Quarters................22

4. Term Structure of GaR for Loosest Decile of FCI ......................................................23

5. Probability Density Functions from Model 3, by FCI Groups and Projection Horizons ....24

6. Term Structures of GaR Model 3 by FCI Groups and Credit Boom Indicator...................25

7. Differences of GaR Term Structures of Loose Minus Mid FCI Groups by Credit Boom...27

8. Term Structures of Predicted Conditional Median and GaR by Initial FCI Groups and

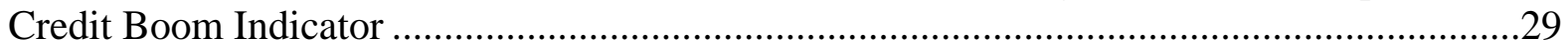

9. Difference of Median and GaR Term Structures for Loose FCI Minus Mid FCI Groups by

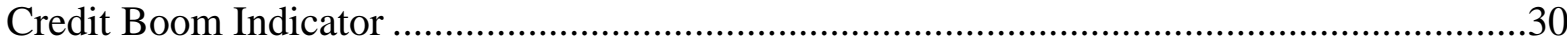

10. Granular Instrumental Variables for Bank Estimated Default Frequency (EDF) Index and

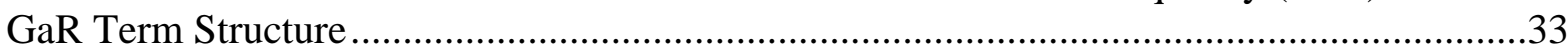

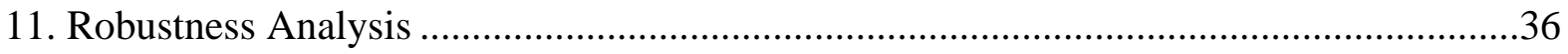


Tables

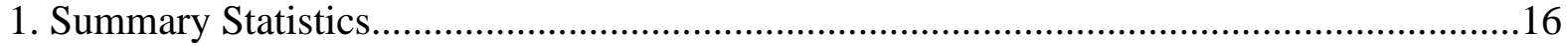

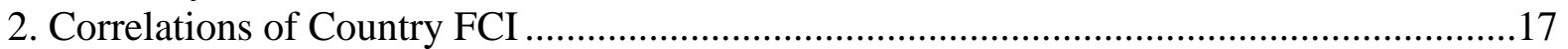

3 Coefficient Estimates for the 5th Percentile of Real GDP Growth ......................................19

4. Coefficient Estimates of Aggregate Bank EDF on GIV ........................................................32

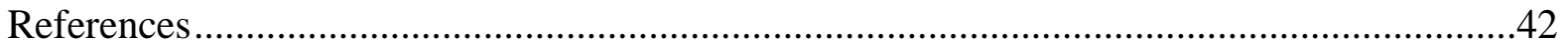

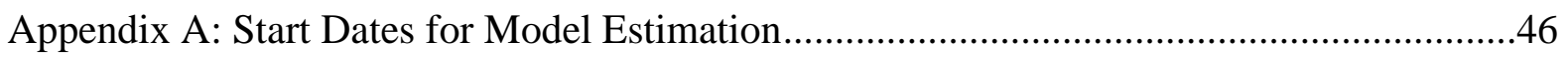

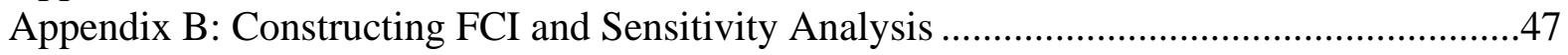

Appendix C: FCI and Credit-to-GDP Growth .....................................................................5

Appendix D: Probability Density Functions from Models 1 and 2, by FCI Groups and

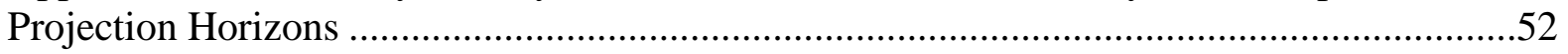

Appendix E: Term Structure of GaR, Model 3, No Lags and Three Lags .............................53 


\section{INTRODUCTION}

Our objective in this paper is to characterize the distribution of forecast GDP growth conditional on financial conditions using a panel of advanced economies (AEs).

Macroeconomic models and forecasting practices focus predominantly on predicting mean growth and ignore volatility or other higher moments of the growth distribution. We show that ignoring these effects may lead to systematic underestimation of downside tail risks when initial financial conditions are loose, especially when credit-to-GDP has been growing rapidly.

In this paper, we estimate the distribution of predicted real GDP growth and evaluate the effects of financial conditions on the median and the lower $5^{\text {th }}$ percentile of the distribution, which we call growth-at-risk (GaR). We construct the term structure of GaR to show how financial conditions affect tail risks for predicted GDP growth at different horizons. We use panel quantile regression methods for our sample of 11 AEs and local projections to forecast out to twelve quarters. ${ }^{2}$ We model empirically the distribution of predicted real GDP growth as a function of financial conditions, credit-to-GDP growth, the interaction of loose financial conditions and high credit-to-GDP growth, in addition to initial output growth and inflation.

Figure 1 shows how the forecasted distribution of GDP growth depends on financial conditions and credit growth. The estimated coefficients on a financial conditions index (FCI) that we construct for the 11 countries, and describe in detail below in Section 3, illustrate the important role of financial conditions for the modeling of the distribution of growth and the implied intertemporal risk-return tradeoff (Figure 1a). ${ }^{3}$ In particular, the coefficient estimates on the FCI differ significantly for the lower $5^{\text {th }}$ percentile and the median of the distribution of GDP growth. The negative coefficients on FCI in near-term quarters ahead for both the $5^{\text {th }}$ percentile and median indicate that the effects of looser financial conditions are to significantly boost expected growth and the GaR value. ${ }^{4}$ But the increase in coefficients over the projection horizon, which is more pronounced for the $5^{\text {th }}$ percentile than the median, highlights the shifting forecasted growth distribution over the

\footnotetext{
${ }^{2}$ The 11 AEs include Australia, Canada, Switzerland, Germany, Spain, France, Great Britain, Italy, Japan, Sweden, and the US.

${ }^{3}$ The FCI for each country is estimated using a vector autoregression model with time-varying parameters following Koop and Korobilis (2014), based on up to 17 price-based variables, including short-term interbank risk spreads, corporate bond spreads, and volatility of stock returns. This approach accounts for differences in data availability of the variables, in contrast to fixed parameters, and we report sensitivity analysis below. The FCI is described more fully in section 3 below, its components are listed in Appendix A, and the FCI series for each country are plotted and results using an alternative index with fixed parameters are reported in Appendix B.
}

${ }^{4}$ We use the term "expected growth" to refer to the forecasted median growth, our measure of central tendency. 
projection horizon. Moreover, the switch in the sign of the estimated coefficients on FCI for the $5^{\text {th }}$ percentile from negative to positive suggests there is an important intertemporal tradeoff associated with financial conditions.

\section{Figure 1. Estimated Coefficients on $\mathrm{FCl}$, Credit Growth, and Credit Boom for Median and 5th Percentile of Real GDP Growth \\ a. $\mathrm{FCl}$

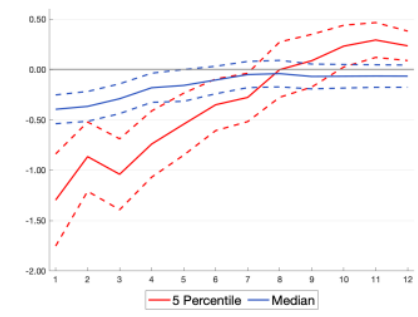 \\ b. Credit-to-GDP growth

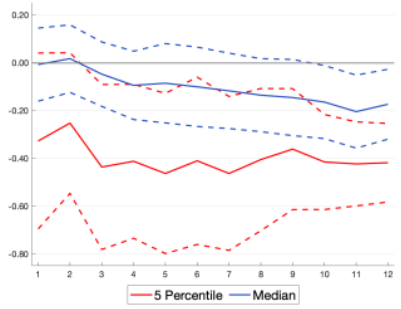 \\ c. Credit boom

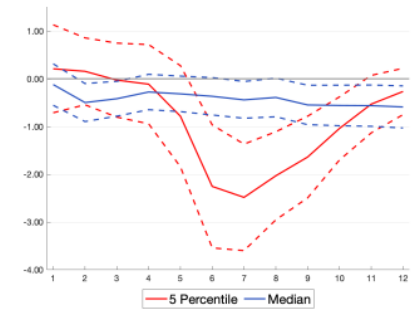 \\ Source: IMF staff estimates. \\ Note: Panel (a) plots the estimated coefficients on the financial conditions index ( $\mathrm{FCl}$ ), (b) plots the estimated coefficients on credit-to-GDP growth, and (c) plots the estimated coefficients on a credit boom indicator, from panel quantile regressions of model 3 with all three variables for the median and the 5 th percentile one to twelve quarters into the future. Real GDP growth (average growth rate for the cumulative period through the quarter at an annual rate) is measured in percent. Higher $\mathrm{FCl}$ represents tighter financial conditions. Credit boom is an indicator variable based on the interaction of loosest three deciles of $\mathrm{FCl}$ and highest three deciles of credit-to-GDP growth. Estimates are based on local projection estimation methods and standard errors are from bootstrapping techniques; bands represent 90 percent confidence intervals. Advanced economies (AEs) include 11 countries with data for most from 1973 to 2017.}

In contrast, the effects of credit-to-GDP growth for the $5^{\text {th }}$ percentile and median of predicted GDP (Figure 1b), while different in magnitude, have a similar pattern over the forecast horizon and indicate the effects of this variable on GDP growth do not vary over time across the quantiles. Figure 1c shows how a credit boom indicator, defined as the combination of loose financial conditions and rapid credit-to-GDP growth, has a significant negative effect for the $5^{\text {th }}$ percentile in quarter five and after, but the effect on the median does not vary. These indicate that loose financial conditions may have a nonlinear effect on GaR which is pronounced in years two and three, but not on median growth. A credit boom thus amplifies the intertemporal tradeoff generated by the reversal in the sign of the FCI coefficients over the projection horizon.

Our empirical specification incorporates findings from other studies of the effects of financial conditions for future output growth. Sharp rises in excess bond premia can predict recessions, consistent with a model of intermediary capital constraints affecting its risk-bearing capacity and thus risk premia (Gilchrist and Zakrajsek, 2012). Also, financial conditions can affect output because financial frictions result in changes in credit supply (see Lopez-Salido, Stein, and Zakrajsek (2017), Mian et al. (2015) and Krishnamurthy and Muir (2020)). Adrian, Boyarchenko, and Giannone (2019) show for the US that financial conditions also affect the 
volatility of the GDP growth distribution, and that there is an inverse relationship between the conditional one-year ahead median and volatility, indicating higher predicted growth is associated with a tighter distribution of growth.

We also allow for an additional effect of FCI on the growth distribution when there is rapid nonfinancial sector credit growth, consistent with research that high borrower credit can be an amplification mechanism. When asset prices and borrower net worth fall, lending spreads rise and borrowing falls disproportionately when there is an external finance premium (Bernanke and Gertler, 1989, and Bernanke, Gertler, and Gilchrist, 1999). In cross-country studies, asset prices and credit growth have been shown to be useful predictors of recessions (Schularick and Taylor, 2012) and significantly weaker recoveries (Jorda, Schularick and Taylor, 2013), and the nonfinancial credit-to-GDP gap is a useful predictor of recessions (Borio and Lowe, 2002). In the US, a large nonfinancial credit-to-GDP gap interacted with financial conditions leads to higher volatility of GDP (Aikman, Lehnert, Liang, Modugno, 2020), and the transmission of monetary policy and financial conditions are affected by credit in the US (Brunnermeier et al. (2017).

Our model estimates indicate a number of important empirical patterns for predicting downside risks to growth. A key result is that GaR for loose FCI is higher in the near-term than in the medium-term, suggesting loose FCI is associated with a tighter growth distribution in the near-term. But the effect changes over the projection horizon. For loose FCI when credit growth is also rapid, GaR values fall substantially from about -0.5 percent in the near-term to less than -2 percent in the medium term. Further, relative to periods when financial conditions are at "average" levels (as defined by the middle four deciles of FCI), the benefits of loose financial conditions in the near-term fade even more quickly. For loose FCI when there is not also rapid credit growth, GaR values fall over the projection horizon, but by less than when credit growth is rapid. The difference suggests a second key result, that high credit can act as an amplification channel of loose financial conditions that leads to greater downside risks.

A third key result is that the greater downside risks to growth in the medium-term from initial loose FCI are not counterbalanced by substantially higher expected growth. While expected growth from loose FCI when credit growth is high is modestly higher than expected growth associated with average initial FCI in the near-term, by about 0.5 percentage points, the difference dissipates and is modestly negative after the first year, while the decline in GaR is much more sharp.

Our interpretation of the time-variation in the distribution of GDP growth reflect changes in the price of risk from initial financial conditions. Changes in the price of risk can arise from financial frictions, such as regulatory capital constraints or VaR models used for risk management, which tie together the price of risk and volatility via the credit supply of 
financial intermediaries (Adrian and Shin, 2014; He and Krishnamurthy, 2012, 2013). If financial conditions initially are loose, constraints are less binding and predicted GDP growth would be higher and its distribution tighter in the near-term. However, the low price of risk and low volatility can contribute to greater risk-taking by financial intermediaries and to higher vulnerabilities, which then leaves the economy prone to a sharper rise in volatility in the event of a negative shock, consistent with the volatility paradox of Brunnermeier and Sannikov (2014).

We report results using a granular instrumental variables (GIV) technique that are consistent with a causal effect of financial conditions on GaR. The GIV approach put forth recently by Gabaix and Koijen (2019) is used to assess whether potential endogeneity bias between financial conditions and GDP growth may be a reason for these empirical results. To apply the GIV method to construct an instrument for financial conditions, we use an expected default frequency (EDF) index for financial firms in place of the FCI because firm-level data, which are necessary for the GIV method, are available. Nonetheless, data constraints on bank-level data limit our estimations to a panel of nine countries starting in 1999. We show that the coefficients on an instrumented financial firm EDF index exhibit the same properties as the coefficients on FCI. That is, the coefficients on the instrumented financial firm EDF index switch signs over the projection horizon and give rise to a similar term structure for $\mathrm{GaR}$ as the FCI. This pattern is consistent with a causal effect of financial conditions on GaR and provides evidence against potential endogeneity bias as a reason for the intertemporal patterns in GaR.

We also report that our primary results based on financial conditions and credit growth are robust to important alternative specifications. We use corporate bond spreads in place of the FCI and find that coefficients on corporate bond spreads show a similar pattern to those for the FCI on the $5^{\text {th }}$ percentile. But the effects of the FCI after corporate bond spreads also are included remain significant, which suggests that variables in the FCI other than corporate bond spreads are relevant to the predicted growth distribution. We also show results are not changed materially if we use a FCI constructed with fixed parameter values rather than timevarying (Appendix B), or add lags of the independent variables (Appendix E). Moreover, for the US, our results of an intertemporal risk tradeoff are robust to controlling for monetary policy, consistent with our results reflecting changes in the price of risk. We also disaggregate nonfinancial credit into nonfinancial business and household credit, but do not find that one type of credit is driving the results. In addition, we evaluate the sensitivity of $\mathrm{GaR}$ estimates to the global financial crisis. The GaR estimates continue to show an intertemporal tradeoff of loose financial conditions when credit growth is high, but the tradeoff of loose financial conditions is weaker, not surprisingly, once observations of significant negative growth are excluded. However, coefficient estimates on FCI for the median and the $5^{\text {th }}$ percentile remain significantly different, with the $5^{\text {th }}$ percentile more responsive to FCI. 
The empirical results in this paper have important implications for macroeconomic models. We document that the forecasted growth distribution changes with financial conditions, a clear violation of a common assumption when estimating macrofinancial models that volatility is independent of growth. Dynamic stochastic general equilibrium models and other models used for policymaking most often focus on impulse response functions that depict conditional growth and, for computational reasons, assume that the mean and variance are independent. However, our results indicate that certainty equivalence is severely violated. Moreover, the covariation of conditional first and higher moments are present at horizons out to twelve quarters. Hence, these results suggest that empirical models of macrofinancial linkages should explore methods to incorporate the endogeneity of higher-order moments and the implications that such endogeneity may have for projections.

The intertemporal tradeoff illustrated by the term structure of $\mathrm{GaR}$ could also have implications for policymaking, although the empirical results are not treatment effects, notwithstanding the granular instrumented variables analysis that provides evidence for causality. A structural model would be needed to evaluate how alternative macroprudential policies could be used to affect $\mathrm{GaR}$. In aspiration, macroprudential policies could aim to tighten financial conditions when conditional expected growth and GaR are relatively high in order to reduce endogenous risk-taking and reduce future financial systemic risks and negative spillovers for the economy. The estimated term structure of GaR conditional on loose versus average initial financial conditions supports the intuition of a tradeoff between building greater resilience in normal times in order to reduce downside risks in stress periods (see Adrian and Liang, 2018). Monetary policy also faces tradeoffs between lower risks to growth in the near-term and greater risks in the medium-term arising from macrofinancial linkages.

A related important benefit of developing a GaR measure is that financial stability risks can be expressed in a common metric that can be used by all macroeconomic policymakers. A common metric can promote greater coordination since alternative policy options can be evaluated on the same terms. It may also improve greater accountability for macroprudential policymakers by providing a metric in terms that are better understood by other policymakers.

This paper builds on Adrian, Boyarchenko, and Giannone (2019), who document that financial conditions can forecast downside risks to GDP growth in the US. We expand it in several ways, by allowing for additional effects of FCI with credit growth, applying the model to a panel of 11 AEs, and extending the forecast horizon beyond the near-term to the medium-term of twelve quarters to study the term structure. We add to recent studies that have found that financial conditions have significant effects on both the mean and higher moments of the distribution, using quantile regressions or other methods (see e.g, Giglio et al. (2016), Coe and Vahey (2020), Kiley (2018) for the unemployment rate, Chavleishvili 
and Manganelli (2019) for the euro area, and Duprey and Ueberfeldt (2020) for Canada. The twelve-quarter projection horizon permits us to explore an intertemporal risk-return tradeoff, as suggested by models of endogenous risk-taking (Brunnermeier and Sannikov, 2014, Adrian and Shin, 2014, Gennaioli and Shleifer, 2018).

Our empirical model can be interpreted within the setting of Adrian and Duarte (2018) where financial frictions in an otherwise standard New Keynesian setting gives rise to time variation in the market price of risk and, as a consequence, in GDP growth. In their model, optimal monetary policy depends on financial conditions in addition to inflation and the output gap because, as in the data, financial conditions predict $\mathrm{GaR}$ in the year-ahead even after controlling for inflation and the output gap. Adrian, Duarte, Grinberg, Mancini-Griffoli (2017) expand this approach to a cross-section of advanced and emerging market economies. Adrian, Duarte, Liang, and Zabczyk (2020) offer an extension to a New Keynesian model to include a financial accelerator and endogenous volatility to match the term structure of GaR, and to develop implications for monetary and macroprudential policies.

The rest of this paper is organized as follows. Section 2 presents the stylized model of GDP growth and financial conditions and describes the quantile regression estimation method, and Section 3 presents the data and estimation of FCI. Section 4 presents estimates of the predicted conditional GDP distribution and the importance of FCIs for the term structure of GaR. Section 5 provides robustness results and Section 6 concludes.

\section{MODELING GROWTH-AT-RISK}

We estimate the dynamics of the GDP distribution over a projection horizon of one to twelve quarters using local projections estimation and apply the model to a panel of 11 AEs. In particular, we estimate conditional distributions of GDP growth for near-term and mediumterm horizons, defined roughly as one-to-four quarters ahead and five-to-twelve quarters ahead, respectively. The 11 AEs represent a set of countries that are defined by the IMF to have systemically important financial sectors and for which we have sufficient data for estimation.

\section{A. Model Estimation with Quantile Regressions}

The estimates of the conditional predicted distribution for GDP growth are from panel quantile regressions. Quantile regressions allow for a general modeling of the functional form of the conditional GDP distribution. We denote $\Delta y_{i, t+h}$ as the annualized average growth rate of GDP for country $i$ between quarters $t$ and $t+h$, and $x_{i, t}$ a vector of conditioning 
variables. ${ }^{5}$ Conditioning variables include current GDP growth, the inflation rate, and financial variables - an index of financial conditions, private nonfinancial credit-to-GDP growth, and a credit boom indicator - all defined below in more detail.

In a panel quantile regression of $\Delta y_{i, t+h}$ on $x_{i, t}$ the regression slope $\delta_{\alpha}^{(h)}$ is chosen to minimize the quantile weighted absolute value of errors

(1) $\hat{\delta}_{\alpha}^{(h)}=\operatorname{argmin} \sum_{i=1}^{\mathrm{N}} \sum_{t=1}^{T-h}\left(\alpha \cdot 1_{\Delta y_{i, t+h}>x_{i, t} \delta}\left|\Delta y_{i, t+h}-x_{i, t} \delta\right|+(1-\alpha) \cdot 1_{\Delta y_{i, t+h}<x_{i, t} \delta}\left|\Delta y_{i, t+h}-x_{i, t} \delta\right|\right)$

where $1_{(\cdot)}$ denotes the indicator function. The predicted value from that regression is the quantile of $\Delta y_{i, t+h}$ conditional on $x_{i, t}$

(2) $\widehat{\boldsymbol{Q}}_{\Delta y_{i, t+h}>x_{i, t}}(\boldsymbol{\alpha})=x_{i, t} \hat{\delta}_{\alpha}^{(h)}$

We then define growth at risk $(\mathrm{GaR})$, the value at risk of future GDP growth, by

(3) $\operatorname{Pr}\left(\Delta y_{i, t+h} \leq \operatorname{GaR}_{i, h}\left(\alpha \mid \Omega_{t}\right)\right)=\alpha$

where $\operatorname{GaR}_{i, h}\left(\alpha \mid \Omega_{t}\right)$ is growth at risk for country $i$ in $h$ quarters in the future at a $\alpha$ probability. Concretely, GaR is implicitly defined by the quantiles of growth rates for a given probability $\alpha$ between periods $t$ and $t+h$ given $\Omega_{t}$ (the information set available at $t$ ). For a low value of $\alpha, \mathrm{GaR}$ will capture the quantiles of growth at the lower end of the GDP growth distribution. That is, there is $\alpha$ percent probability that growth would be lower than GaR. We define GaR to be the lower $5^{\text {th }}$ percentile of the GDP growth distribution. We show below estimates of the full probability density function, which illustrate that the choice of 5 percent as the cutoff is a reasonable representation of the lower tail.

To track how the conditional distribution of GDP growth evolves over time, we use Jorda's (2005) local projection method. This allows us to also explore how different states of the economy can potentially interact with FCIs in nonlinear ways in forecasting the GDP

\footnotetext{
${ }^{5}$ We estimate conditional quantile regressions (CQR), although we note the evolving literature on so-called 'unconditional' quantile regressions (UQR). Originally proposed by Firpo, Fortin and Lemieux (2009), application of UQR is conducive to inference in cross-sectional regression settings to understand, for instance, the effect of a marginal change in workers' characteristics on each quantile of the overall distribution of individuals' wages. In such a case, unconditional quantiles of two individuals with different education levels would be their wage quantiles among all individuals in the population. In our case, given we seek to generate forecast distributions corresponding to a single target variable (GDP growth) over time conditional on a set of determinants, rather than information pertaining to a set of distinct individuals, CQR is the appropriate framework.
} 
growth distribution at different time horizons (see Jorda (2005) and Stock and Watson (2018)), while at the same time having a model that does not impose dynamic restrictions embedded in VAR models. Note that the approach intends to capture the forecasting effects of FCIs on GDP growth distribution, not causal effects, although we provide below some empirical support for causal effects. For simplicity, we will refer to the former as "effects" in the discussion that follows.

We estimate the model in a panel regression with country fixed effects. Estimation of the panel quantile regressions with quantile-specific country fixed effects is feasible when the panel structure has $T$ (the time series dimension) much larger than $N$ (number of countries), as is the case in our forecasting application (Galvao and Montes-Rojas, 2015, and Cech and Barunik, 2017). ${ }^{6}$ Inferential procedures based on bootstrap resampling with such a panel quantile set-up is considered in Galvao and Montes-Rojas (2015). These authors build on the so-called $(y, x)$-pairs bootstrap (Freedman, 1981) under which entire rows of data (containing the dependent and conditioning variables) are sampled with replacement, and demonstrate asymptotic feasibility under various assumptions for relative sizes $N$ and $T$.

Specifically, in our application we resample rows of data from the temporal dimension of each country, keeping unchanged the cross-sectional structure of the panel. To account for temporal dependence present in the data, we use a block-bootstrap (Lahiri, 2003, and Kapetanios, 2008). This consists of resampling 'blocks' formed of contiguous rows of data. ${ }^{7}$ In the analysis below, we generate bootstrap standard errors considering block widths of 4 , 6 and 10 quarters, but report only block widths of 4 quarters as results with alternative specifications are quite similar. All standard errors estimates are based on 10,000 bootstrap samples.

Below we generally report the direct estimates from the quantile regressions for the $5^{\text {th }}, 50^{\text {th }}$, and $95^{\text {th }}$ percentiles, rather than estimates from a smoothed distribution. However, we also show probability density functions which we recover by mapping the quantile regression estimates into a skewed t-distribution, following Adrian et al. (2019), which allows for four time-varying moments - conditional mean, volatility, skewness, and kurtosis. To do so, we fit the skewed $t$-distribution developed by Azzalini and Capitaion (2003) in order to smooth the quantile function:

\footnotetext{
${ }^{6}$ The literature to date on estimating panel quantile regressions with fixed effects has focused mostly on the problem where the number of cross-sectional units $N$ far exceeds $T$ (Koenker, 2004). In general, estimation and associated asymptotic properties are based on restricting fixed effects to be invariant across different quantiles (Canay, 2011).

7 This assumption that errors are uncorrelated across countries is not unusual. It would be difficult to change in our estimations because country-level data do not have uniform availability, and we have unbalanced panels.
} 
(4) $f(y ; \mu, \sigma, \theta, v)=\frac{2}{\sigma} d T\left(\frac{y-\mu}{\sigma} ; v\right) T\left(\theta \frac{y-\mu}{\sigma} \sqrt{\frac{v+1}{v+\frac{y-\mu}{\sigma}}} ; v+1\right)$

where $d T(\cdot)$ and $T(\cdot)$ respectively denote the PDF and CDF of the skewed $t$-distribution. The four parameters of the distribution pin down the location $\mu$, scale $\sigma$, fatness $\nu$, and shape $\theta$. We use the skewed $t$-distribution as it is a flexible yet parametric specification that captures the first four moments.

\section{B. Financial Conditions and Credit-to-GDP Growth}

We present three model specifications, where $x_{i, t}$ includes (1) current GDP growth, inflation, FCI, and a constant, (2) variables in (1) plus private nonfinancial credit-to-GDP growth, and (3) variables in (2) plus a credit boom dummy variable, defined by the interaction of loose FCI and high credit-to-GDP growth. In particular, we define credit boom as a dummy variable in country $i$ in period $t$ as:

(5) Credit boom $_{i, t}=$

$\left\{1\right.$ if $\mathrm{FCI}_{i, t}$ is in the loosest 3 deciles of $\mathrm{FCI}_{i}$ and (credit-to-GDP growth) ${ }_{i, t}$ is in the highest decile of (credit-to-GDP) ${ }_{i}$ 0 else

Credit-to-GDP growth is measured over the previous eight quarters. The joint condition of loose financial conditions and rapid credit-to-GDP growth helps to exclude periods when credit-to-GDP growth has been high mainly because it is starting to reverse from a bust and while FCIs are still near recession tightness, since those conditions would not be consistent with a credit boom. ${ }^{8}$

Coefficients on the credit boom indicator variable that are negative would be consistent with the effect of financial conditions through macrofinancial linkages on output growth. When there is high vulnerability because of indebted households and businesses and a low price of risk, the combination increases the likelihood of financial instability if the price of risk rises. Highly-indebted borrowers not only see their net worth fall when the price of risk rises and asset prices fall, but the decline is more likely to leave them underwater and more likely to default and a pullback in credit. ${ }^{9}$ Moreover, a steep decline in net worth and a sharp decline

\footnotetext{
${ }^{8}$ We choose the bottom three deciles for FCI to simplify the presentation below of the GaR term structures conditioned on initial FCIs by deciles. The results are robust to using similar alternative thresholds, like the bottom two deciles or one-third of the FCI distribution, but the dummy variable would then cross over deciles and complicate the presentation.

${ }^{9}$ The addition of credit growth also helps to address the possibility that the estimated effects of FCI on the conditional distribution of GDP growth may simply reflect the different speeds at which financial conditions and GDP growth respond to common negative shocks, where FCIs might incorporate news more quickly than the real economy. According to this argument, FCIs do not predict GDP growth, but FCI and GDP growth are correlated because of a common shock. However, if the effects of loose FCIs on GDP growth also depend on
} 
in aggregate demand could put the economy in a liquidity trap or deflationary spiral. That situation would be seen in the data as lower downside risk (higher $\mathrm{GaR}$ ) in the near-term, but higher downside risk to GDP (lower GaR) in the medium-term.

Our empirical model aims to capture the dynamics following loose financial conditions, allowing for nonlinearities. To fix ideas, changes in the distribution of GDP growth are generated by changes in the price of risk, which is measured via financial conditions. Loose financial conditions can lead to a buildup of vulnerabilities in the presence of financial frictions, such as capital requirements or VaR models of financial institutions. When asset prices rise and financial conditions loosen, increased net worth can make regulatory constraints for financial intermediaries less binding, leading to a reduction in risk premia (He and Krishnamurthy, 2013) and additional risk-taking (Adrian and Shin, 2014). Loose financial conditions may also ease constraints for borrowers, who then can accumulate excess credit because they do not consider negative externalities for aggregate demand (see, for example, Korinek and Simsek, 2016).

In addition, lower risk premia may be associated with exuberant sentiment, and periods of compressed risk premia can be expected to be followed by a reversal of valuations (Greenwood and Hanson, 2013). Lopez-Salido, Stein, and Zakrajsek (2017) show that periods of narrow risk spreads for corporate bonds and high issuance of lower-rated bonds are useful predictors of negative investor returns in the subsequent two years. The negative returns lead to lower growth, likely from a pullback in credit supply, providing empirical evidence of an intertemporal tradeoff of current loose financial conditions at some future cost to output. A lower price of risk and greater risk taking can be a result of beliefs that extrapolate the past and that neglect downside risks (Gennaioli and Shleifer, 2018). Extrapolation can explain why the price of risk can be very low for prolonged periods, and neglect of downside risks can explain how financial systems can become highly leveraged as agents lever up when they share beliefs that the price of risk is unlikely to increase sharply and that other agents are somehow protected from negative shocks.

\section{DATA}

Quarterly data for real GDP growth and consumer price indexes (CPI) to measure inflation (year-to-year percent change) for the 11 countries are available from the International

high credit growth, the nonlinear results would be more consistent with models of endogenous risk-taking and amplification of shocks, rather than just different adjustment periods to a common shock. For a common shock, we would not expect that the predictive power of a low price of risk should be stronger with the presence of higher credit growth. The significance of the credit boom variable also supports the GIV results presented below. 
Financial Statistics (IFS). ${ }^{10}$ Nonfinancial credit-to-GDP ratios are from the BIS, where nonfinancial credit is the sum of household and business credit.

We construct FCIs for each of the 11 countries using up to 17 price-based variables. ${ }^{11}$ The FCI captures domestic and global financial price factors, such as short-term funding spreads, corporate bond risk spreads, equity prices and volatility, and banking sector expected default frequencies (EDFs). Data availability varies across the countries, and the starting dates for each of the data series and the start date for the model estimation by country is shown in Appendix A.

Following a common practice in constructing indices, the FCIs are estimated with factor methods. In particular, we follow Koop and Korobilis (2014) methodology that combines the estimation methodology of Primiceri's (2005) time-varying parameter vector autoregression (TVP-FAVAR) model and dynamic factor model of Doz, Giannone, and Reichlin (2011). With this model we estimate FCI as an unobserved common factor for the set of 17 financial variables described above. This approach has two benefits relative to fixed-parameter models: (i) it allows for dynamic interactions between the FCIs and macroeconomic conditions which can evolve over time, and (ii) it allows for differences in starting dates for some financial indicators with a flexible estimation procedure. ${ }^{12}$ In Appendix B we provide details of the TVP-FAVAR model and results with a fixed-parameter factor (FP-FAVAR) model.

Summary statistics of the model variables for the panel of eleven countries are presented in Table 1. Values in the tables are averages across countries and across time. The values represent the sample estimation periods starting in 1975, 1980, or 1981 for most, except for

\footnotetext{
${ }^{10}$ Estimates of potential growth for the 11 countries are not available on a consistent basis, or for the full sample periods.

${ }^{11}$ The variables include interbank spreads, corporate spreads, sovereign spreads, term spreads, equity returns, equity return volatilities, equity implied volatilities, changes in real long-term rates, interest rate implied volatilities, house price returns, the percent changes in the equity market capitalizations of the financial sectors to total market capitalizations, equity trading volumes, expected default frequencies for banks, market capitalizations for equities, market capitalizations for bonds, domestic commodity price inflation rates, and foreign exchange movements.

${ }^{12}$ There are two alternative solutions to the unbalanced data availability. First, one could replace missing observations with zeros and estimate an FCI via a fixed-parameter factor model (FP-FAVAR). This alternative would risk putting too little weight on series that are important and only become available later in the sample. Second, one could restrict the FCI estimation using a FP-FAVAR to the period in which all series all available. But that would severely restrict our sample and we want to include as many business and credit cycles as possible (see Appendix C). Moreover, both approaches would impose constant weights to relationships between financial variables and the macroeconomy that may be changing over time (see Koops and Korobilis 2014). Appendix B compares our FCI using TVP-FAVAR and compares its results relative to a FP-FAVAR where missing observations are replaced by zeros.
} 
Spain which starts in 1992 (see Appendix A). The roughly 40-year sample period for most of the countries allows us to capture multiple business and credit cycles, rather than only the global financial crisis.

Table 1. Summary Statistics

\begin{tabular}{|l|c|c|c|c|c|c|}
\hline & Mean & Std Dev & Median & 10th Percentile & 90th Percentile & $\mathbf{N}$ \\
\hline GDP growth & 2.221 & 3.446 & 2.454 & -1.607 & 5.932 & 1600 \\
\hline Inflation & 3.565 & 3.425 & 2.620 & 0.364 & 8.582 & 1600 \\
\hline FCI & -0.011 & 1.038 & 0.013 & -1.378 & 1.175 & 1600 \\
\hline $\begin{array}{l}\text { Credit-to- } \\
\text { GDP growth }\end{array}$ & 0.543 & 1.068 & 0.450 & -0.663 & 1.831 & 1600 \\
\hline Credit boom & 0.079 & 0.269 & 0.000 & 0.000 & 0.000 & 1600 \\
\hline
\end{tabular}

Source: IMF International Financial Statistics database: Bank for International Settlements; and IMF staff estimates.

Note: Table includes descriptive statistics for 11 AEs: Australia, Canada, Switzerland, Germany, Spain, France, Great Britain, Italy, Japan, Sweden, and the US. The start of the estimation period is either 1975 or 1980 for most of the advanced economies. Specific starting dates for each country are shown in Appendix A.

For our sample, average annual real growth is 2.2 percent and inflation is 3.6 percent. The average credit-to-GDP ratio is 1.34 , and the eight-quarter moving average growth in the ratio is 0.54 percent, indicating credit tended to grow faster than GDP through the period. Periods when the credit boom is equal to 1 are 8.0 percent of sample.

The constructed FCI is standardized and the mean and median values are near 0 . The correlations of FCI and its key components, such as the corporate bond spread and stock return volatility, vary across the countries. As shown in Table 2, average correlations across countries of the FCI with the interbank spread, corporate spread term spread, and stock return volatility range are relatively high, 0.57 to 0.68 . In some countries, the correlations of FCI with the interbank spread and corporate spread are higher than 0.80 . In contrast, the average correlation with the term spread is only 0.19 . 
Table 2. Correlations of Country FCl with Selected Components

\begin{tabular}{|l|c|c|c|c|c|c|}
\hline $\begin{array}{l}\text { Correlation } \\
\text { of FCI with: }\end{array}$ & Mean & Std dev & Median & Min & Max & $\begin{array}{c}\text { N } \\
\text { countries }\end{array}$ \\
\hline $\begin{array}{l}\text { Interbank } \\
\text { spread }\end{array}$ & 0.57 & 0.24 & 0.58 & -0.08 & 0.84 & 11 \\
\hline $\begin{array}{l}\text { Corporate } \\
\text { spread }\end{array}$ & 0.66 & 0.23 & 0.69 & 0.08 & 0.89 & 11 \\
\hline Term spread & 0.19 & 0.2 & 0.24 & -0.29 & 0.39 & 11 \\
\hline $\begin{array}{l}\text { Equity return } \\
\text { volatility }\end{array}$ & 0.68 & 0.09 & 0.7 & 0.48 & 0.82 & 11 \\
\hline
\end{tabular}

Source: IMF International Financial Statistics database: Bank for International Settlements; and IMF staff estimates.

Note: Table shows summary statistics of the correlation of country $\mathrm{FCl}$ and selected country financial variables for $11 \mathrm{AEs}$ : Australia, Canada, Switzerland, Germany, Spain, France, Great Britain, Italy, Japan, Sweden, and the US. The start of the FCI sample period is either 1975 or 1980 for most of the advanced economies. Specific starting dates for each country are shown in Appendix A.

Regression estimates (not shown) show that FCIs have significant coefficients for credit-toGDP growth multiple quarters ahead, suggesting credit-to-GDP growth increases in response to loose financial conditions with a lag. Charts of FCI and credit-to-GDP growth for the 11 countries are in Appendix C. These data indicate that the coefficient estimates do not reflect a single episode of loose financial conditions and a credit boom but reflect a number of different business and credit cycles.

\section{EMPIRICAL RESULTS}

In this section, we first present quantile regression coefficients for the $5^{\text {th }}$ percentile for models 1 to 3 . The coefficients on FCI on the $5^{\text {th }}$ percentile of the GDP growth distribution change over the projection horizon, and this result is robust to adding credit growth and a credit boom indicator. Next, we show the time series of GaR averaged across countries at a given projection horizon as well as the median and $9^{\text {th }}$ percentile, and show there is greater variance in downside than in upside risks. Third, we show the probability density functions of forecasted growth for models 1 to 3 , which illustrate the increase in the negative skew between the short-term and the medium-term arises primarily from loose financial conditions, shown by estimates of model 1 , and are amplified by a credit boom, shown by estimates of model 3.

Next, we examine based on model $3 \mathrm{GaR}$ term structure conditional on initial financial conditions and credit boom indicator, where GaR is calculated for each country-time observation for $h=1$ to 12 . We document that GaR values based on initial loose financial 
conditions are higher in the near-term but decline in the medium term, and the decline is greater if initial credit-to-GDP growth. We then look also at the projected conditional median growth by initial FCI groups to illustrate a potential intertemporal risk-return tradeoff from initial loose financial conditions. The estimates show that while initial loose FCI and a credit boom project higher expected median growth and GaR in the near-term, the median differential declines modestly while the decline in the GaR differential is substantial, suggesting sharp increases in downside risks without the benefit of higher growth.

We present some evidence that the effect of FCI has a causal effect on the growth distribution using a GIV method. These results are compelling but limited because of limited data availability over time, and to some extent across countries. Finally, we discuss a set of robustness tests, several of which are reported in detail in appendixes to this paper.

\section{A. Quantile Regression Estimates}

Table 3 presents coefficient estimates for the $5^{\text {th }}$ percentile of the GDP growth distributions for model specifications 1,2, and 3; we show estimates for only the even-numbered projection horizons $h$ to simplify the table. Model 1includes current GDP growth, inflation, and FCI. Estimated coefficients for FCI, where lower FCI values represent looser financial conditions, are negative and significant in the near-term, but positive and significant in later quarters. The change in coefficients over the forecast horizon indicate that looser FCI is associated with a higher value for the $5^{\text {th }}$ percentile (a tighter predicted growth distribution) through quarter 6 , but then a lower $5^{\text {th }}$ percentile value after quarter 8 , indicating greater downside risks in year three. The coefficients on GDP growth are positive and significant in the near-term and diminish to zero after $h=8$, while the coefficients on inflation are negative and significant in the near-term and similarly diminish to zero over time.

Model 2 adds credit-to-GDP growth and model 3 also adds the credit boom indicator variable. The coefficients on credit-to-GDP growth in model 2 are negative and significant starting at $\mathrm{h}=4$ and remain that way over the horizon. Importantly, the coefficients on FCI remain significant and still show the reversal in signs over the projection horizon. In model 3, the coefficients on credit boom are large and significant around $\mathrm{h}=6$ to 10 quarters, indicating the effect of an initial credit boom substantially increases downside risk (reduces GaR) in the second year. The coefficients on credit-to-GDP growth remain negative and significant, and the coefficients on FCI still indicate a reversal. The coefficients on GDP growth and inflation do not change materially in models 2 and 3 from model 1 . 
Table 3. Coefficient Estimates for the $5^{\text {th }}$ Percentile of Real GDP Growth Model 1: FCI, GDP Growth, Inflation

\begin{tabular}{|c|c|c|c|c|c|c|}
\hline & $h=2$ & $h=4$ & $h=6$ & $h=8$ & $h=10$ & $h=12$ \\
\hline \multirow[t]{2}{*}{$\mathrm{FCl}$} & $-0.908^{* \star *}$ & $-0.757^{* \star *}$ & $-0.329 *$ & 0.247 & $0.324^{* * *}$ & $0.231^{* * *}$ \\
\hline & $(0.206)$ & $(0.206)$ & $(0.163)$ & $(0.163)$ & (0.119) & $(0.083)$ \\
\hline \multirow[t]{2}{*}{ GDP growth } & $0.319 * * *$ & $0.190 * * *$ & $0.109^{*}$ & $0.083^{*}$ & $0.069^{*}$ & 0.007 \\
\hline & $(0.074)$ & $(0.053)$ & $(0.057)$ & $(0.042)$ & $(0.035)$ & $(0.025)$ \\
\hline \multirow[t]{2}{*}{ Inflation } & $-0.331^{* * *}$ & $-0.323^{* * *}$ & $-0.254^{\star * *}$ & $-0.116^{*}$ & -0.061 & $-0.086^{*}$ \\
\hline & $(0.095)$ & $(0.078)$ & $(0.089)$ & $(0.065)$ & (0.059) & $(0.048)$ \\
\hline
\end{tabular}

Model 2: Model 1 with Credit-to-GDP Growth

\begin{tabular}{|c|c|c|c|c|c|c|}
\hline & $h=2$ & $\mathrm{~h}=4$ & $h=6$ & $h=8$ & $h=10$ & $h=12$ \\
\hline $\mathrm{FCl}$ & $\begin{array}{l}-0.864^{\star * *} \\
(0.205)\end{array}$ & $\begin{array}{l}-0.751^{* * *} \\
(0.201)\end{array}$ & $\begin{array}{l}-0.345^{\star *} \\
(0.156)\end{array}$ & $\begin{array}{l}0.159 \\
(0.169)\end{array}$ & $\begin{array}{l}0.335^{\star * *} \\
(0.120)\end{array}$ & $\begin{array}{l}0.279 * * * \\
(0.082)\end{array}$ \\
\hline GDP growth & $\begin{array}{l}0.316^{\star \star \star} \\
(0.072)\end{array}$ & $\begin{array}{l}0.217^{\star \star \star} \\
(0.052)\end{array}$ & $\begin{array}{l}0.105^{*} \\
(0.052)\end{array}$ & $\begin{array}{l}0.040 \\
(0.040)\end{array}$ & $\begin{array}{l}0.039 \\
(0.029)\end{array}$ & $\begin{array}{l}0.021 \\
(0.024)\end{array}$ \\
\hline Inflation & $\begin{array}{l}-0.322^{\star \star \star} \\
(0.091)\end{array}$ & $\begin{array}{l}-0.261^{\star * \star} \\
(0.080)\end{array}$ & $\begin{array}{l}-0.173^{*} \\
(0.089)\end{array}$ & $\begin{array}{l}-0.057 \\
(0.062)\end{array}$ & $\begin{array}{l}-0.064 \\
(0.042)\end{array}$ & $\begin{array}{l}-0.045 \\
(0.032)\end{array}$ \\
\hline Credit-to-GDP growth & $\begin{array}{l}-0.252 \\
(0.157)\end{array}$ & $\begin{array}{l}-0.419^{\star *} \\
(0.168)\end{array}$ & $\begin{array}{l}-0.705^{\star * *} \\
(0.217)\end{array}$ & $\begin{array}{l}-0.705^{\star \star *} \\
(0.189)\end{array}$ & $\begin{array}{l}-0.535^{\star * *} \\
(0.117)\end{array}$ & $\begin{array}{l}-0.457^{* * *} \\
(0.096)\end{array}$ \\
\hline
\end{tabular}

Model 3: Model 1 with Credit-to-GDP Growth and Credit Boom

\begin{tabular}{|c|c|c|c|c|c|c|}
\hline & $h=2$ & $h=4$ & $h=6$ & $h=8$ & $h=10$ & $h=12$ \\
\hline \multirow[t]{2}{*}{$\mathrm{FCl}$} & $-0.864^{\star \star \star}$ & $-0.739 * * \star$ & $-0.349 * *$ & -0.000 & $0.233^{*}$ & $0.236^{\star *}$ \\
\hline & $(0.209)$ & $(0.200)$ & $(0.156)$ & $(0.167)$ & $(0.126)$ & $(0.089)$ \\
\hline \multirow[t]{2}{*}{ GDP growth } & $0.316^{* * *}$ & $0.217^{* * *}$ & $0.148^{* \star *}$ & 0.060 & $0.051^{*}$ & 0.019 \\
\hline & $(0.074)$ & $(0.052)$ & $(0.052)$ & $(0.040)$ & $(0.030)$ & $(0.024)$ \\
\hline \multirow[t]{2}{*}{ Inflation } & $-0.322^{\star * *}$ & $-0.273^{* * *}$ & $-0.277^{* * *}$ & $-0.166^{\star *}$ & $-0.094^{* *}$ & -0.054 \\
\hline & $(0.092)$ & $(0.082)$ & $(0.089)$ & $(0.064)$ & $(0.044)$ & $(0.036)$ \\
\hline \multirow[t]{2}{*}{ Credit-to-GDP growth } & -0.252 & $-0.412^{\star \star}$ & $-0.410^{*}$ & $-0.405^{\star *}$ & $-0.415^{\star \star \star}$ & $-0.418^{\star \star \star}$ \\
\hline & $(0.179)$ & $(0.196)$ & $(0.213)$ & $(0.181)$ & $(0.121)$ & $(0.100)$ \\
\hline \multirow[t]{2}{*}{ Credit boom } & 0.158 & -0.111 & $-2.252^{* * *}$ & $-2.030^{* * *}$ & $-1.039 * *$ & -0.264 \\
\hline & $(0.426)$ & (0.504) & $(0.783)$ & (0.561) & (0.402) & (0.295) \\
\hline
\end{tabular}

Source: IMF staff estimates.

Note: Table reports estimated coefficients for the 5th percentile of real GDP growth from panel quantile regressions and local projection methods, and standard errors are from block boot-strapping techniques. Real GDP growth (average growth rate for the cumulative period through the quarter at an annual rate) is measured in percent. Higher $\mathrm{FCl}$ represents tighter financial conditions. Credit-to-GDP growth is measured over the past eight quarters. Credit boom is an indicator variable based on the interaction of loosest three deciles of $\mathrm{FCl}$ and highest three deciles of credit-to-GDP growth. Sample is 11 Advanced economies with data for most from 1973 to 2017 . Models include country indicator variables. ${ }^{* *} p<0.01$, ${ }^{* *} \mathrm{p}<0.05,{ }^{*} \mathrm{p}<0.1$. 
The addition of credit variables does not change the pattern of coefficients on the FCI for the $5^{\text {th }}$ percentile, which are negative in the near-term and become positive in quarters further out. The coefficients provide strong empirical support for an intertemporal tradeoff of loose financial conditions and low downside risk at short horizons, which set the stage for a deterioration in performance two to three years later.

The significant coefficients for the credit boom indicator are consistent with macrofinancial linkages that can lead to greater tail risks for predicted growth. Otherwise, it could just be that financial conditions are forward-looking and respond quickly to adverse events, whereas it takes time for such events to work their way through real economic activity. If the link from financial conditions to growth were just a common shock, we would not also expect larger costs because growth in credit is higher. The higher costs in the medium term estimated for high credit growth periods is consistent with an endogenous risk-taking channel helping to explain the reduction in volatility in the near-term, which allows more risk-taking, and leads to higher volatility in the medium-term.

\section{B. Time Series of Average GaR}

Figure 2 shows the time series of predicted GaR estimates (averaged across countries) from model 3 at the projection horizon of four quarters $(h=4)$. Also plotted are the conditional median and the $95^{\text {th }}$ percentile for $\mathrm{h}=4$, as well as realized growth (shifted forward by four quarters). The time series reveals that lower projected median growth is associated with lower GaR, consistent with conditional growth and volatility being negatively correlated. In sharp contrast, there is very little variability at the $95^{\text {th }}$ percentile, suggesting greater variability arises from greater downside risk than upside risk. 
Figure 2. Predicted Average Conditional Distribution of Real GDP Growth Four-Quarters Ahead

(In Percent)

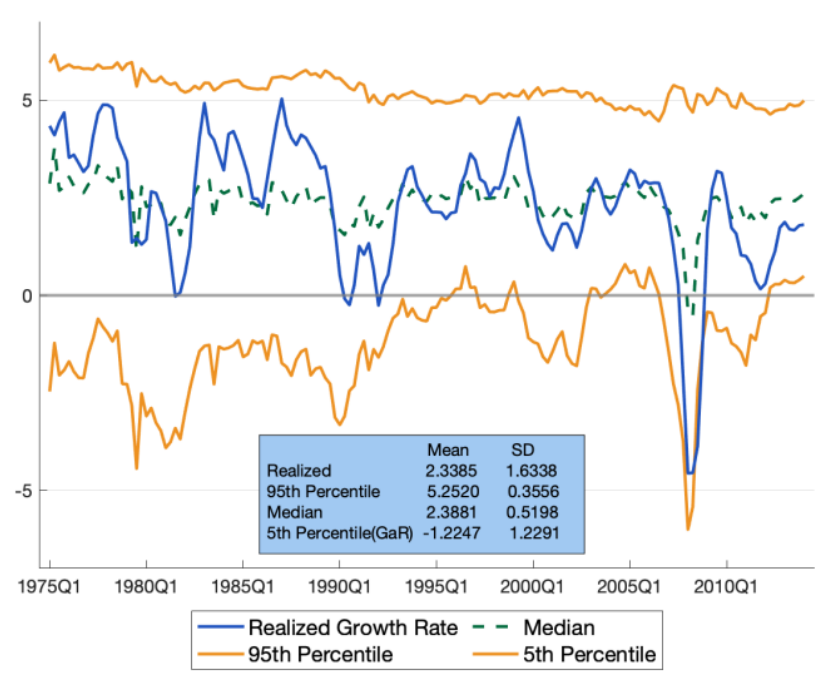

Source: IMF staff estimates.

Note: Figure plots the cross-country averages of predicted conditional mean, 5th percentile (GaR), and 95th percentile of real GDP growth from model 3 panel quantile regressions. Real GDP growth is measured in percent. Advanced economies include 11 countries with data for most from 1973 to 2017.

In particular, the mean GaR for AEs over the sample period is -1.22 percent, with a standard deviation of 1.23 , whereas the standard deviation of the $95^{\text {th }}$ percentile is lower at 0.36 , even though the mean $95^{\text {th }}$ percentile is much higher, at 5.25 percent. Basically, the conditional $95^{\text {th }}$ percentile shows little variation, while GaR is highly variable. The downside risk as represented by GaR shows much greater variability than upside risk as the conditional mean changes over time. These results extend Adrian et al. (2019) to a panel of AEs.

\section{Probability Density Functions of Forecasted Growth and GaR}

In this section, we show the predicted probability density functions (pdfs) from fitting the quantile regression estimates to a skewed- $t$ distribution, as described above by equation (4). The growth distributions are shown in Figure 3 for models 1, 2, and 3, each at $\mathrm{h}=4,8$, and 12, conditional on the decile of loosest financial conditions. The differences across models help to highlight the separate contributions of FCI and the credit-to-GDP variables, and the differences across $\mathrm{h}$ illustrate the intertemporal tradeoff of initial loose financial conditions. 


\section{Figure 3. Probably Density Functions from Models 1, 2, and 3 for Decile of Loosest FCl, by Projection Quarters}

a. Model 1 , loose $\mathrm{FCl}$, by $\mathrm{h}$

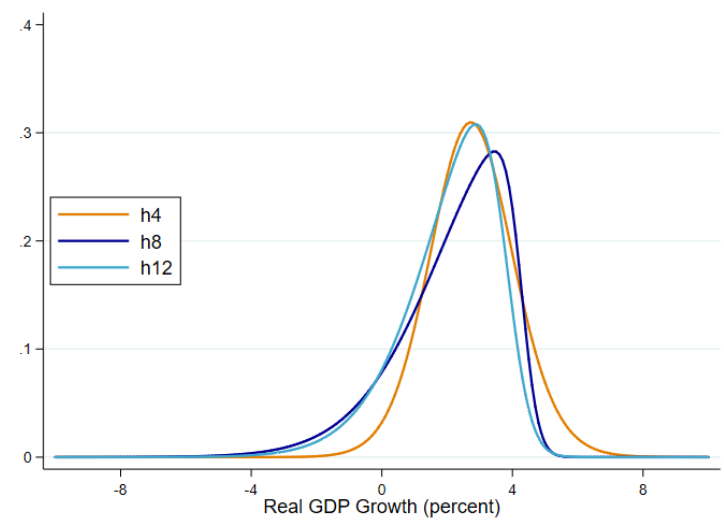

c. Model 3, loose FCl, Credit Boom, by h

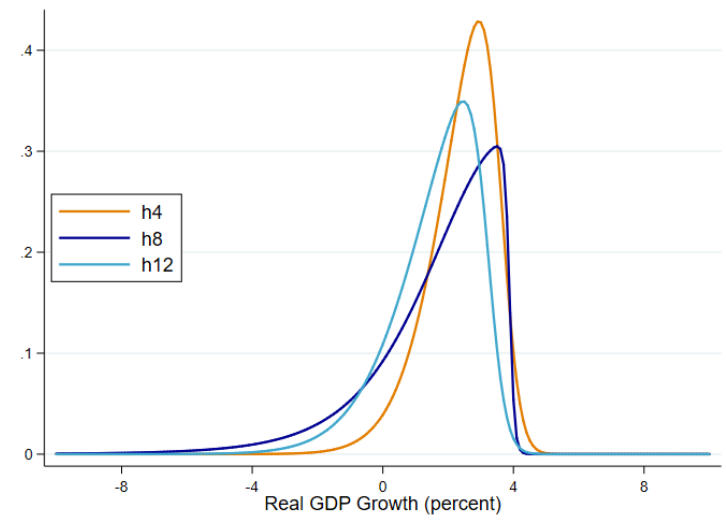

b. Model 2, loose FCl, by $\mathrm{h}$

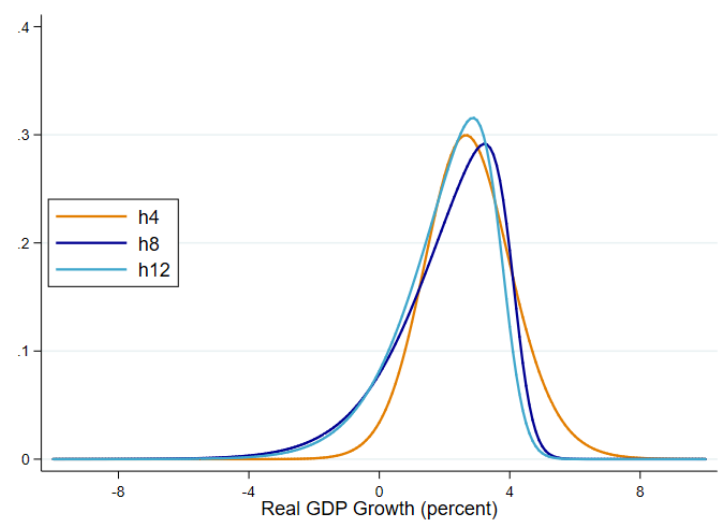

d. Model 3, loose FCl, Not Boom, by h

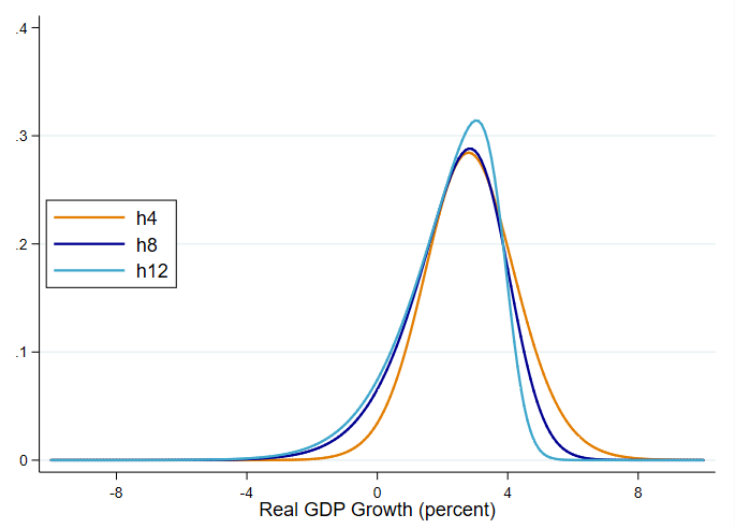

Source: IMF staff estimates.

Note: Probability density functions are estimated using panel quantile regression methods and fitted to a skewed $t$ distribution and are shown for loose $\mathrm{FCl}$ (bottom decile) by credit boom indicator for h equal to 4, 8, and 12 quarters. Advanced economies include 11 countries most with data from 1973 to 2017.

The pdfs for model 1 show a relatively tight real GDP growth distribution at $\mathrm{h}=4$, but the left tail of the distribution widens significantly over the forecast horizon and becomes more leftskewed at $\mathrm{h}=8$. The $5^{\text {th }}$ percentile is lower at $\mathrm{h}=8$ and $\mathrm{h}=12$ than at $\mathrm{h}=4$. These pdfs illustrate the intertemporal tradeoff of loose financial conditions, with a tighter distribution in the nearterm but greater downside risks in the medium-term. The pdfs for model 2 which includes credit-to-GDP growth look very similar to results from model 1, indicating that while the coefficients on credit-to-GDP growth are significant, the magnitude of its effects on the predicted GDP growth distribution are not substantial in most periods. 
The pdfs for model 3 are shown separately for the credit boom indicator equal to 1 or 0 . It is evident that a credit boom has a significant effect on the shape of the predicted distributions, tighter at $h=4$ and wider at $h=8$, consistent with high credit-to-GDP growth as an amplification channel. The changes in the distributions over $h$ when there is not a credit boom, while not dramatic, importantly still illustrate the intertemporal tradeoff associated with loose FCI, such as observed in model 1 without any credit variables.

To illustrate more clearly the model differences for $\mathrm{GaR}$, we plot the predicted $5^{\text {th }}$ percentiles for each model for $\mathrm{h}=1$ to 12 , conditioned on the loose FCI decile for each country and GDP and inflation in that situation (Figure 4a). There is little notable difference in the term structures of GaR for models 1 and 2, and both show GaR values are high in the near term and then fall sharply. For model 3 with a credit boom, the negative effects on downside risk are most evident in quarters beginning in the second year out, but the effects dissipate. Not surprisingly, for model 3 with loose financial conditions but without a credit boom, the downside risks are not as prominent. Nonetheless, the values are significant and positive in the near term, $h=3$ to 6 , and are negative and significant $h=9$ to 12 . Moreover, the difference between the GaR values with a credit boom and without are significantly different from zero for h from 5 to 11 (Figure 4b).

\section{Figure 4. Term Structure of GaR for Models 1, 2, and 3 for Loosest Decile of FCl}

a. Gar for loose $\mathrm{FCl}$, by model

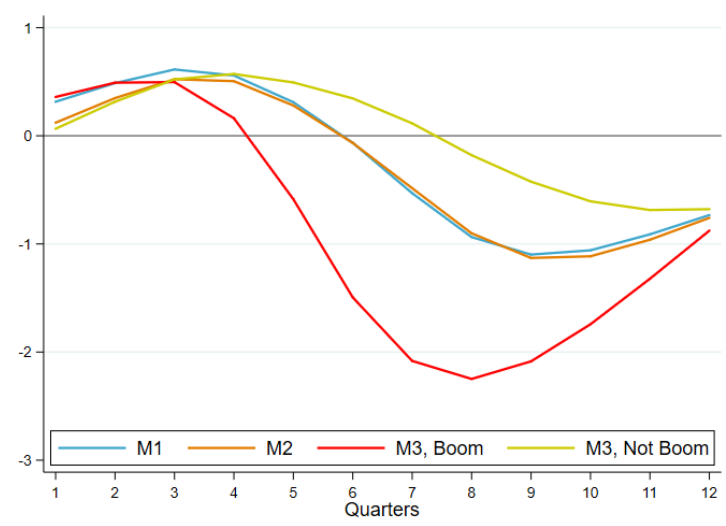

b. Difference in GaR, model 3, credit boom or not

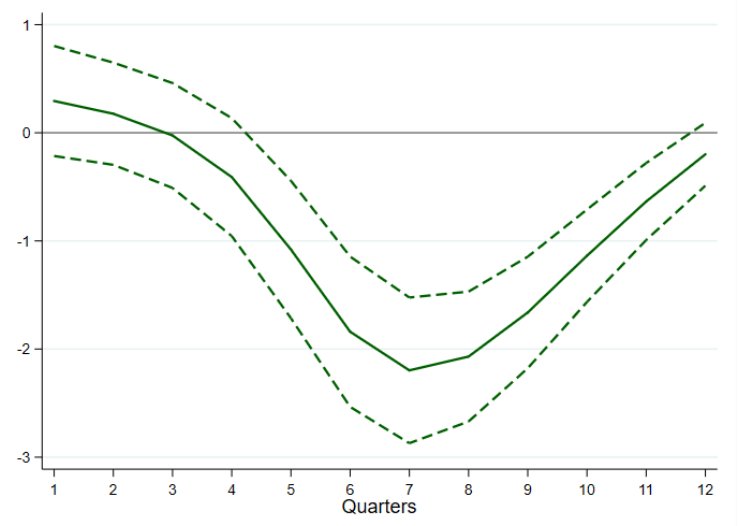

Source: IMF staff estimates.

Note: Figures plot the GaR (projected growth at the 5th percentile) at an annual rate for initial $\mathrm{FCl}$ in the loosest decile for alternative model specifications. Real GDP growth, vertical axis, is measured in percent. Estimates are based on quantile regressions with local projection estimation methods and standard errors are from bootstrapping techniques. Advanced economies include 11 countries with data for most from 1973 to 2017. 
Next, we turn to results for a broader set of initial FCI groups. In Figure 5, we add the pdfs for the tightest FCI decile and a mid FCI, approximated by the middle four deciles, to those already shown for the loosest FCI decile. We focus on model 3 and show two different time horizons. (Results for models 1 and 2 are shown in Appendix D.)

Figure 5. Probability Density Functions from Model 3, by FCl Groups and Projection Horizons

a. $\quad$ Model 3, Credit Boom, $\mathrm{h}=4$

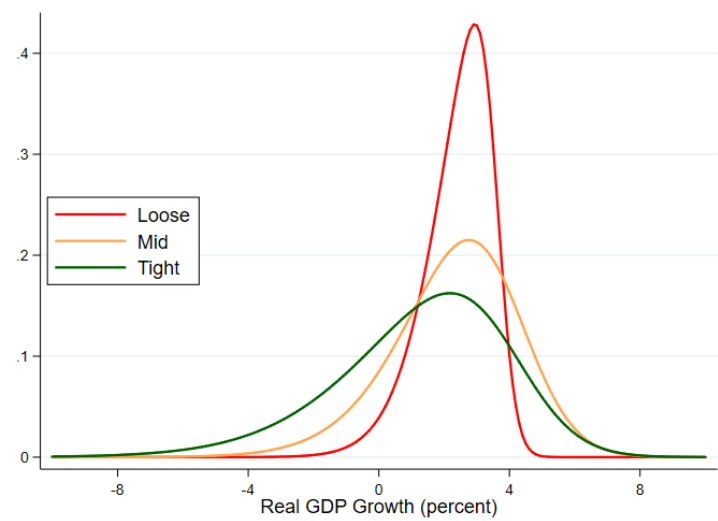

c. Model 3, Not Boom, $\mathrm{h}=4$

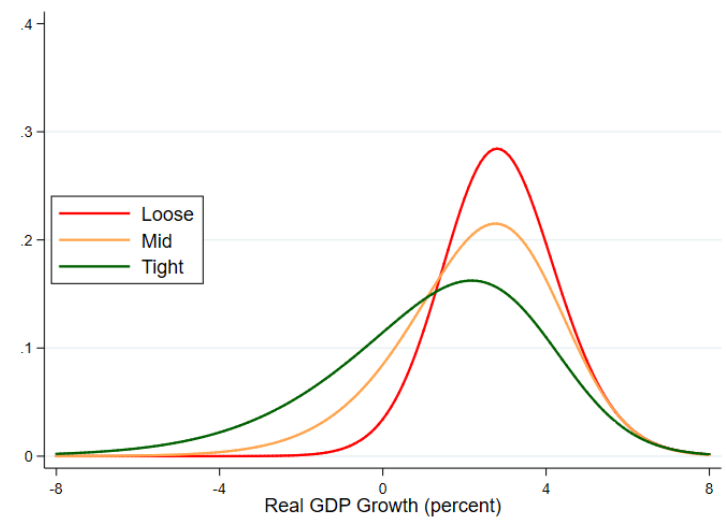

b. Model 3, Credit Boom, $\mathrm{h}=12$

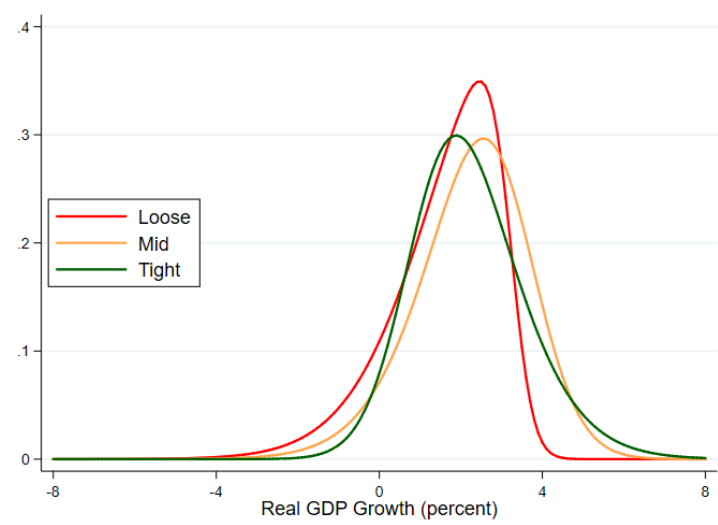

d. $\quad$ Model Not Boom, $h=12$

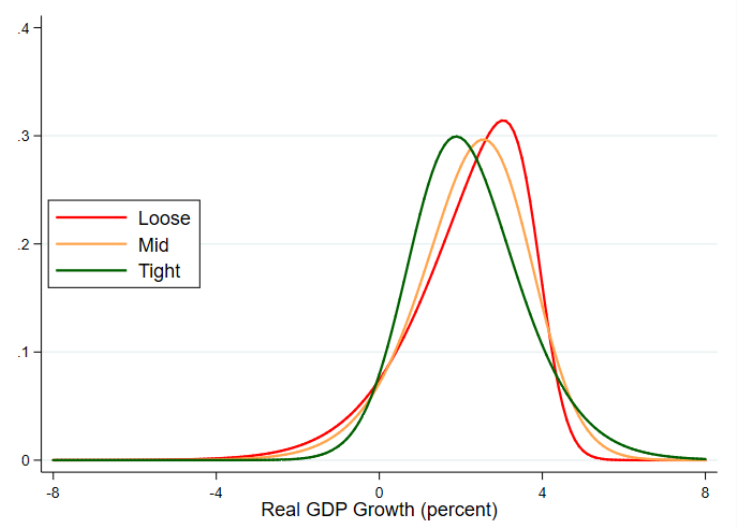

Source: IMF staff estimates.

Note: Probability density functions shown are estimated based on model 3 using panel quantile regression methods and fitted to a skewed t distribution, and are shown for the loosest decile, the tightest decile, and mid (middle four deciles) of $\mathrm{FCl}$, for $\mathrm{h}=4$ and $\mathrm{h}=12$ quarters. Advanced economies include 11 countries most with data from 1973 to 2017.

In near-term horizons around $\mathrm{h}=4$, the predicted GDP growth distributions for the mid FCI and tight FCI groups are much wider than for the loose FCI group. By the end of the forecast horizon at $\mathrm{h}=12$, however, the distributions based on mid FCI and tight FCI have tightened, 
while the distribution for loose FCI has widened, consistent with an amplification channel for rapid credit growth when FCIs are loose.

Overall, these distributions indicate substantial shifts and increased downside risks from $h=4$ to $h=12$ when initial financial conditions are loose in a credit boom. For loose FCI but without a credit boom, the distribution also shifts, but the shift is less pronounced. ${ }^{13}$

\section{Term Structures of GaR by Initial FCI Groups}

The probability density functions shown in Figure 5 provide the entire smoothed distribution for a given FCI, credit boom indicator, and projection horizon. Next, we focus on risks in the lower tail, specifically the $5^{\text {th }}$ percentile, although the density functions indicate that results would be robust to other percentiles in the near vicinity, such as the $2.5,7.5$, or $10^{\text {th }}$ percentiles. For the $5^{\text {th }}$ percentile, we plot the term structure of GaR from model 3 based on a range of initial FCI groups, by whether credit boom is equal to one or zero (Figure 6).

\section{Figure 6. Term Structures of GaR Model 3 by FCl Groups and Credit Boom Indicator}

a. Credit boom

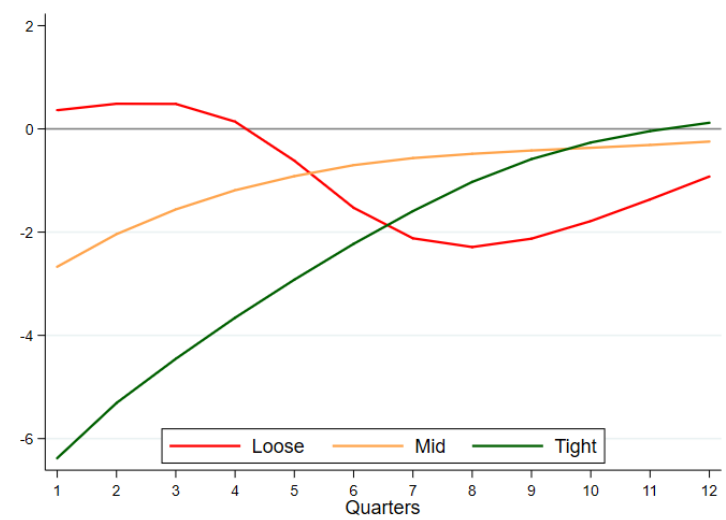

b. No credit boom

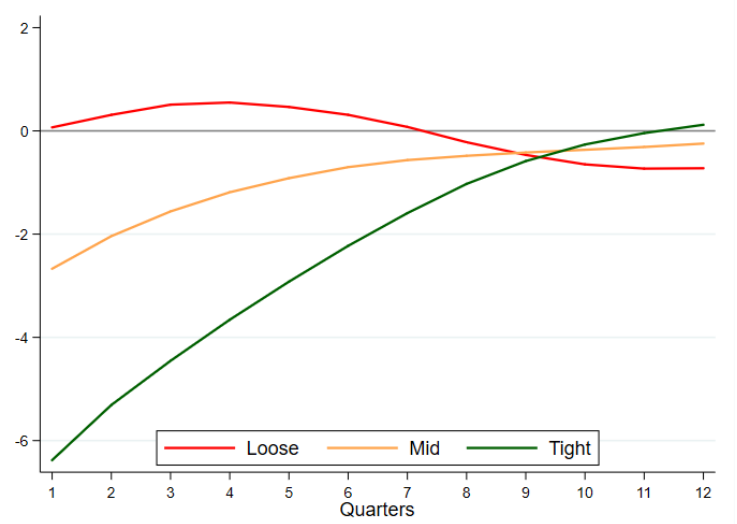

Source: IMF staff estimates.

Note: Figures plot the GaR (projected growth at the 5th percentile) at an annual rate. Real GDP growth, vertical axis, is measured in percent. The GaR projections are grouped on initial loosest decile, tightest decile, and mid (middle four deciles). Estimates are based on quantile regressions with local projection estimation methods. Advanced economies include 11 countries with data for most from 1973 to 2017.

The term structures indicate an intertemporal tradeoff for downside risk when initial FCIs are loose, and the tradeoff from loose FCI is more substantial if credit growth is rapid. We

\footnotetext{
${ }^{13}$ We can also express the changes in distributions over the projection horizon into the probability of GaR falling below zero (not shown). The probability in the near-term is negligible but rises significantly to almost 20 percent in the medium-term for loose FCI and a credit boom. Without a credit boom, the probability of negative growth rises more modestly from zero to about 9 percent for loose FCI.
} 
demonstrated earlier that the tradeoff can arise from FCI only, but the presence of a credit boom amplifies the fall in the $5^{\text {th }}$ percentile values of the distribution. When initial FCIs are loose (in the bottom decile), the estimated GaRs are initially high and then fall over most of the projection horizon, indicating downside risks increase in the medium-term; the downward slope is much sharper when there is also a credit boom (Figure 6a) relative to when there is not (Figure 6b). Specifically, GaR is about -0.5 percent in the near-term for Loose FCI and credit boom, but it then falls significantly over the projection horizon to less than -2.0 percent at around $h=8$, a swing of about 2.5 percentage points. As shown in Figure $4 \mathrm{~b}$ above, the differences in GaR conditional on a credit boom are significantly different from when there is not a credit boom.

Estimated GaRs for initial mid FCI rise initially and then level out at about -0.5 percent in the medium-term. That is, the term structure for the typical moderate FCI group slopes upward rather than downward, as moderate FCIs do not increase downside risks to growth in the medium-term. ${ }^{14}$

To compare the differences in the GaR term structures based on initial FCI, we calculate the differences between the loose FCI and the mid FCI, and we test for the statistical difference between the term structures by calculating standard errors by bootstrapping the differences in GaRs at each horizon $h$. The differences in the term structures between the average GaR based on loose FCI with a credit boom and average GaR based on mid FCI are positive and statistically significant, based on 90 percent confidence interval, in the near-term and turn negative and statistically significant in the medium-term (Figure 7a), indicating that the lower downside risks in the near-term from the loose FCI reverse and become larger in quarters further out. The difference in term structures for Loose and mid FCI groups for no credit boom is also positive and significant in the short-term, and falls over the projection horizon, but the magnitude of the decline is smaller (Figure 7b). Under credit boom conditions, the difference in GaRs is about 2 percentage points lower at around $h=8$ to 10 than when no credit boom, suggesting credit growth plays an important role in amplifying changes in financial conditions, consistent with theories of macrofinancial linkages.

\footnotetext{
${ }^{14}$ Note that because credit boom was defined by high credit growth and FCI in the bottom three deciles, the estimated term structures of $\mathrm{GaR}$ for the Mid40 do not differ for credit boom and not credit boom.
} 


\section{Figure 7. Differences of GaR Term Structures of Loose Minus Mid FCI Groups by Credit Boom}

a. Credit boom

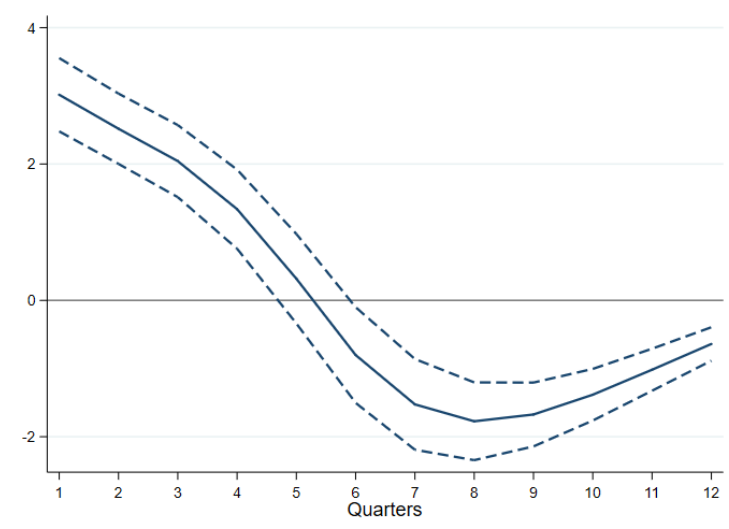

b. No credit boom

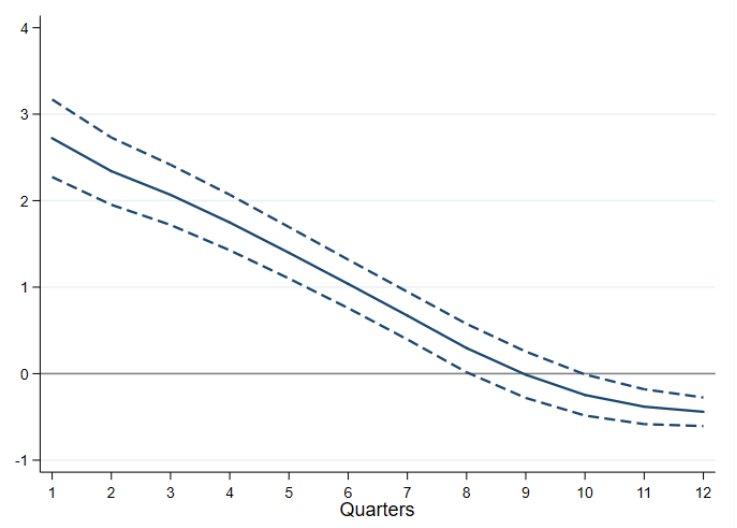

Source: IMF staff estimates.

Note: Figures plot the differences in the GaR term structures of the Loose minus the Mid FCl groups. Difference in real GDP growth, vertical axis, is measured in percent. Standard errors are from bootstrapping techniques on the differences. Advanced economies include 11 countries with data for most from 1973 to 2017.

Returning to the term structures in Figure 6, the estimates also show that the worst outcomes in the short run are when FCIs are initially extremely tight, in the top decile. GaR for this decile is very low in the short-run (less than -6 percent), suggesting the economy is predicted to be in a deep recession or a financial crisis. However, the large downside risks dissipate over time and converge in the medium term to about the same GaR as for initial moderate financial conditions. We view very tight FCIs as reflecting the realization of a negative shock, which leads to a sharp tightening of FCIs, not a deliberate policy choice. What determines initial financial conditions is outside this empirical model, but a number of models with endogenous risk-taking behavior would predict that loose FCIs that also lead to greater financial vulnerabilities set the stage for sharper tightenings in FCIs once a negative shock occurs because of fire sales (Brunnermeier and Pedersen (2009), Brunnermeier and Sannikov (2014), and Adrian and Shin (2014)). Or sharper tightenings in FCI may reflect sharp sentiment reversals that are triggers that interact with vulnerabilities and lead to recessions and credit busts (Minsky, 1977 and Genaoili and Shleifer, 2018). We leave to future work an approach to estimating the term structures of the joint distribution between FCIs and GDP growth.

\section{E. Term Structures of Predicted Median Growth and GaR by Initial FCI Groups}

So far, we have focused on GaR, the lower $5^{\text {th }}$ percentile of the forecasted growth distribution. But a drop in the $5^{\text {th }}$ percentile from initial loose FCIs could also be 
accompanied by higher expected growth, in which case an alternative interpretation of higher growth and higher risk is possible. In this section, we evaluate the projected additional expected growth and reduction in downside risks from initial loose financial conditions relative to typical financial conditions over the term structure. We find that the forecasted additional expected growth conditional on loose FCI falls modestly over the projection horizon. That is, conditioning on loose FCI relative to mid FCI, the intertemporal risk tradeoff - less risk now at the cost of more risk later - is not mitigated by higher expected growth later.

To see this tradeoff, we plot the projected median and GaR term structures for the loose and mid FCI groups, with and without a credit boom (Figures 8a and 8b). First, median growth is a bit higher in the near-term for FCI in the loose than for mid FCI in both cases, and then falls over the projection horizon. That is, the contribution to growth from loose FCI diminishes over the projection horizon. Second, GaR is higher (downside risk is lower) for FCI in the loose than for mid in the near-term, but it then falls over the projection horizon. The reversal is substantial for credit boom conditions. Note also that the projected median growth for mid FCI is flat over the projection horizon, at slightly under 2 percent, suggesting this FCI group is a reasonable characterization of neutral financial conditions, and that neutral financial conditions are consistent with steady growth and diminishing downside risks. 


\section{Figure 8. Term Structures of Predicted Conditional Median and GaR by Initial FCI Groups} and Credit Boom Indicator

a. Credit boom

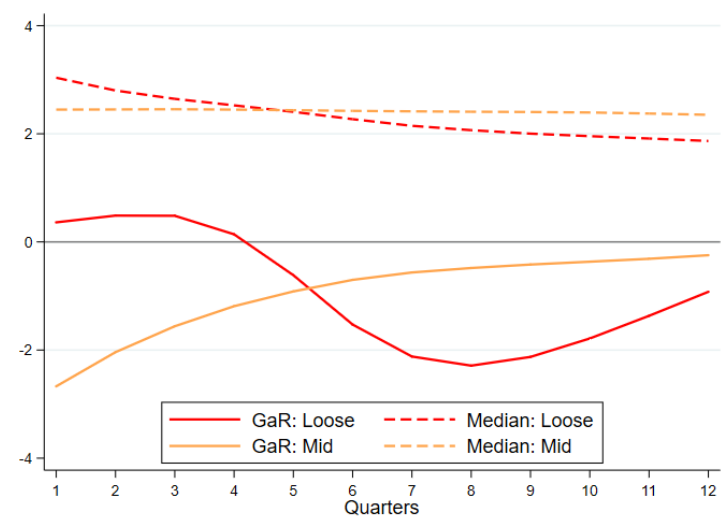

b. No credit boom

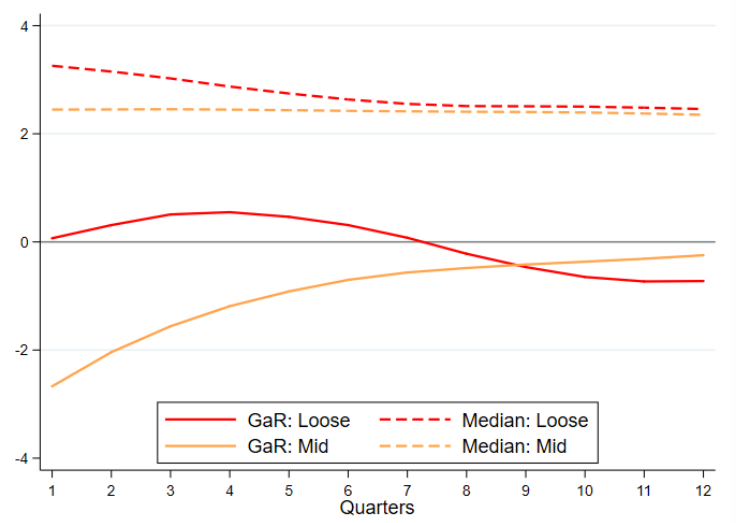

Source: IMF staff estimates.

Note: Figures plot projected median and GaR (projected growth at the 5th percentile) at an annual rate for initial FCl levels Loose and Mid. Real GDP growth, vertical axis, is measured in percent. Estimates are based on quantile regressions with local projection estimation methods, and standard errors are from bootstrapping techniques. Advanced economies include 11 countries with data for most from 1973 to 2017.

Figure 9 plots the information in Figure 8 as differences in the term structures between the bottom decile and the neutral case for the projected medians and GaR. The differences make it more evident that the decline in GaR is much steeper than the decline in the median growth for the loosest FCI group relative to the mid FCI group. This configuration also illustrates the costs of a credit boom. In contrast, when there is not a credit boom, the decline in GaR - the amplification effect - is less sharp, and the decline in the any potential boost to growth is very modest. 


\section{Figure 9. Difference of Median and GaR Term Structures for Loose FCI Minus Mid FCI Groups by Credit Boom Indicator}

a. Credit boom

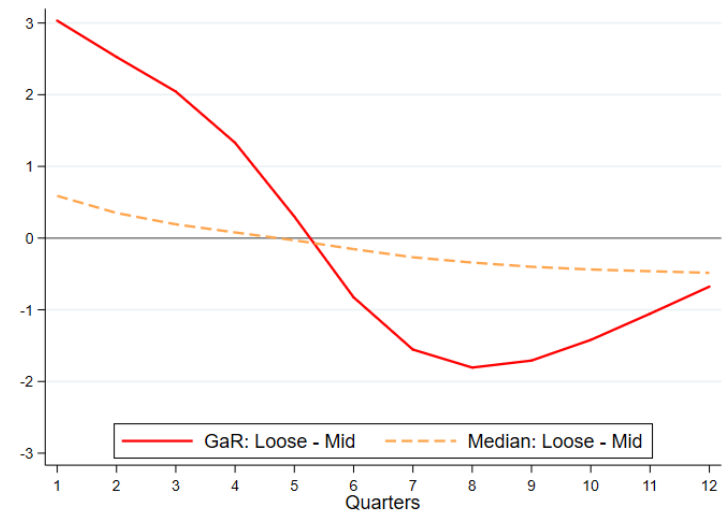

b. No credit boom

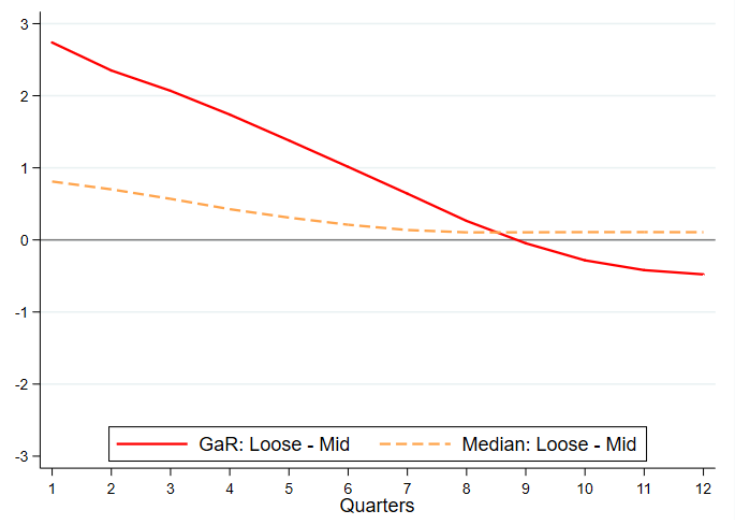

Source: IMF staff estimates.

Note: Figures plot the differences in the projected median and GaR (projected growth at the 5th percentile) at an annual rate for initial FCl levels Loose and Mid. Difference in real GDP growth, vertical axis, is measured in percent. Estimates are based on quantile regressions with local projection estimation methods, and standard errors are from bootstrapping techniques. Advanced economies include 11 countries with data for most from 1973 to 2017.

\section{F. Applying Granular Instrumental Variables to Evaluate Causality}

In this section, we evaluate a possible causal effect of financial conditions on GaR by using the Granular Instrumental Variable (GIV) methodology (Gabaix and Koijen, 2019). ${ }^{15}$ This evaluation would address a concern that the FCI could reflect shocks more quickly than real variables such as GDP growth, and the empirical patterns we estimate simply reflect the delay. To implement this, we approximate our broad index of financial conditions with an index of banking firms' expected default frequencies (EDFs), a market-based measure of credit risk based on a distance to default model. ${ }^{16}$ Like financial conditions indexes and

\footnotetext{
15 This analysis is related but distinct from the question of whether financial conditions can predict GDP growth and distribution once real conditions are incorporated. Plagborg-Moller, et al, 2020 study if financial variables provide any marginal forecasting power once a global indicator, which is already based on financial variables and real variables is included. Our analysis addresses a separate but related question, whether financial conditions affect GDP growth and tail risks, not whether financial conditions are a better forecasting tool after accounting for their concurrent interactions with real activity indicators. Financial conditions can be causal if they reflect frictions in financial markets, such as risk management constraints, that affect the supply of credit.

${ }^{16} \mathrm{EDF}$ is a measure of the probability that a firm will default over a specified period of time. "Default" is defined as failure to make scheduled principal or interest payments. According to the Moody's EDF model, a
} 
corporate bond spreads, EDFs are viewed to have predictive power for economic growth and risks to growth. We use EDFs as an alternative measure for financial conditions because we can obtain firm-specific EDFs which are necessary to construct an instrument to use the GIV method. ${ }^{17}$

The GIV method exploits the variability of the underlying bank-level data and its heterogeneous size distribution to construct a valid instrumental variable for aggregate data. When there are agents that are large enough (that is, they are "granular"), their idiosyncratic shocks will have an aggregate impact. Gabaix and Koijen show that this distribution allows a valid and optimal instrumental variable to be constructed for the aggregate data. The exclusion restriction is that aggregate shocks to each country's aggregate bank EDF index are uncorrelated with idiosyncratic shocks to individual banks' EDFs.

The bank-level EDFs are from Moody's and are calculated based on a firm's assets, liabilities, and the market value and volatility of its equity. We use bank market capitalization also from Moody's to construct each bank's share in the country-level EDF. Importantly, these shares show an important degree of concentration, which is needed for the GIV approach to be valid. We start with the earliest data available in 1999:Q4, and drop the firms for which the data are insufficient, either because it starts late or the firm disappears from the sample). We are left with only two banks in each France and Spain, so we drop these countries from the sample for these estimations. The resulting sample includes nine countries for which we have at a minimum of six firm-level EDFs covering the whole period, with five of those countries having many more firms and representing complex and larger financial systems.

The GIV method relies on recovering idiosyncratic shocks for individual financial institutions EDFs by filtering common factors. We apply the GIV methodology in the following way. ${ }^{18}$ First, for each country we use the individual bank EDFs and market capitalization shares and construct an aggregate bank EDF index. Second, we apply principal component analysis (PCA) to the individual series and use an optimal number of principal components. Third, the resulting idiosyncratic errors from the PCA estimation and market capitalization shares are then used to construct the GIV as in equation (24) in Gabaix and

firm will default when the market value of its assets (the value of the ongoing business) falls below its liabilities payable (the default point).

${ }^{17}$ Note that individual financial firm level measures for comparable corporate bond spreads, for example, are very difficult to get for the countries in our sample.

${ }^{18}$ This application of GIV to a panel with local projections follows closely Aldasoro et al. (2019). The main difference is that here we estimate a quantile panel rather than a model for the conditional mean. 
Koijen. ${ }^{19}$ Finally, we use the GIV to instrument the aggregate EDF indexes in the panel quantile estimation with local projections for GDP growth (equation (1)), where EDF replaces FCI. Because EDF data are available for a shorter time period than FCI, starting in 1999:Q4 rather than in the mid-1970s, we estimate our model for a shorter time period and focus on model specification 1.

The estimations show that the instrument GIV EDF is relevant, with a first-stage F-test of 29 and the coefficient on the GIV is significant at 1 percent level (Table 4). Next, we show the estimated coefficients for instrumented EDF along with the bank EDF index for the $5^{\text {th }}$ percentile of GDP growth (Figure 10a). ${ }^{20}$ The estimated coefficients for the bank EDF index on the $5^{\text {th }}$ percentile are significant and show the same pattern as when estimating the model based on FCI, and the estimated coefficients for the instrumented EDF index exhibit a very similar pattern. These results for the instrumented EDF provide strong evidence against endogeneity bias and in favor of causality; that is, looser financial conditions (lower EDF) have a causal effect in the compression of downside risks in the near term and then an increase in downside risks in the medium term. Moreover, the estimated effects suggest that the non-causal effects have an attenuation bias in quarters further out, the outer horizon, as the estimated coefficients for the $5^{\text {th }}$ percentile are substantially greater when the EDF is instrumented than when it is not.

Table 4. Coefficient Estimates of Aggregate Bank EDF on Granular Instrumental Variable

\begin{tabular}{|c|c|}
\hline & First stage \\
\hline EDF GIV & $0.439^{* \star}$ \\
& $(-0.088)$ \\
\hline Observations & 567 \\
\hline R-squared & 0.136 \\
\hline Countries & 9 \\
\hline
\end{tabular}

Source: IMF staff estimates.

Note: Coefficient estimates of aggregate bank EDF on granular instrumental variable (GIV) from bank-level EDF for nine countries, 1999:Q4 to 2015:Q4. The first-stage panel estimation includes GDP growth, inflation, and a country indicator. $* \star * \mathrm{p}<0.01$.

\footnotetext{
${ }^{19}$ We take a conservative stance on the appropriate number of factors and use the ones with eigenvalues above the unit.

${ }^{20}$ Because of the much shorter sample period, we do not include credit-to-GDP growth and the credit boom indicator as the resulting estimation for those variables becomes too noisy.
} 


\section{Figure 10. Granular Instrumental Variables (GIV) for Bank Estimated Default Frequency (EDF) Index and GaR Term Structure}

a. Coefficients for bank EDF index and instrumented EDF index on the 5th percentile

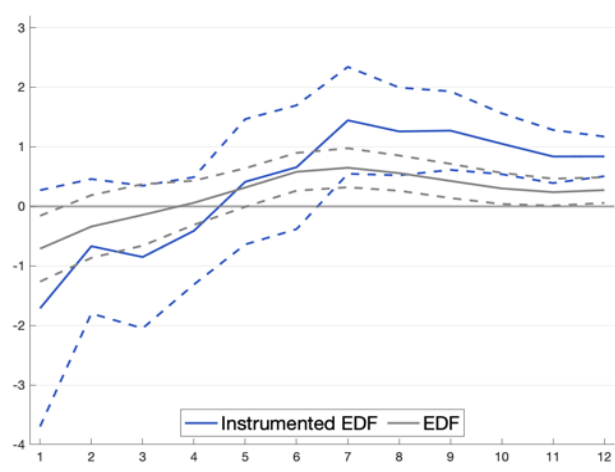

b. Term structure of GaR based on GIV EDF estimations for model 1, by EDF groups

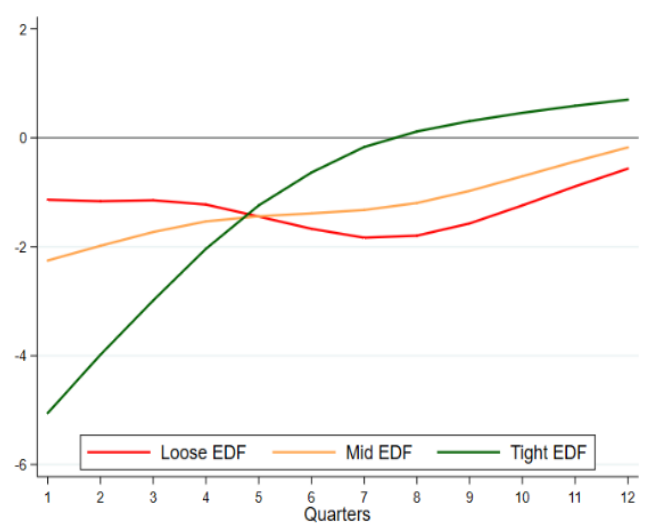

Source: IMF staff estimates.

Note: Panel (a) shows the estimated coefficients on the bank EDF index on the 5th percentile, and the estimated coefficients on the instrumented bank EDF based on granular instrumental variables method. Panel (b) shows the constructed term structure of GaR from model 1 estimates based on the instrumented bank EDF, by EDF deciles representing the lowest, highest, and middle group. Data start in 1999:Q4 given limited data availability for firm-specific EDFs needed to construct the instrument, and exclude two of the eleven countries in our full sample because there were not enough banks to construct the instrument.

Figure 10b shows the resulting term structures of GaR for different initial EDF groups from the GIV method. The results show a decrease in GaR for the low EDF group over the projection horizon, similar to the pattern based on loose FCI for model 1 shown in Figure 4a, though the GaR values decline to about -2 percent, rather than -1 percent. These differences may reflect differences in the samples and estimation periods, but likely also reflect that FCI is affected by factors not captured in bank EDFs, but for which we cannot instrument.

\section{G. Interpreting the Intertemporal Risk-Return Tradeoff}

We have shown with GaR and the probability density functions that the differences in term structures between loose and moderate initial FCI groups are statistically different. While we do not model the determination of FCIs and our estimates are not treatment effects, notwithstanding the results from the GIV method above, the increased downside risks in the medium-term associated with looser financial conditions (lower price of risk) suggests that policymakers might want to consider intertemporal risk tradeoffs. 
An important consideration, conditional on this intertemporal tradeoff, is whether the higher future downside risks are substantial enough to want to forego the slightly higher expected median growth and lower downside risks in the near-term. We have not specified a policymaker's welfare function, as our goal in this paper is to test empirically for whether a tradeoff exists. A welfare function that would apply a simple time discount factor might not find the future higher downside risks to be great enough to offset the near-term benefits of lower downside risks.

But a more economically significant tradeoff might exist if the welfare function were to incorporate that the costs of large downside risks are high. For example, recessions can lead to permanent losses in output, rather than a temporary decline with a rebound back to trend, and recessions with banking crises have greater losses (Cerra and Saxena, 2008). The costs of recessions in which there are large-scale job losses and financial distress are viewed to be costly and associated with significant waste because worker separations may destroy contractually fragile relationships (Hall, 1995; Ramey and Watson, 1997). Costs may also increase with the severity of the recession, which often are greater when there is also a banking crisis or other financial crisis. Reinhart and Rogoff (2009) and Schularick and Taylor (2012) document that recessions with financial stress are much more costly and may take five to eight years to return to pre-crisis levels, several years longer than recoveries following normal recessions. Wolfers (2003) finds that greater macroeconomic volatility and higher unemployment has an adverse impact on different social welfare metrics.

Another case where higher downside risks in the future might be more costly than implied by a time discount factor is if policymakers have limited tools to remedy a recession if one were to occur. This could be the case if monetary policy rates are near the zero-lower bound, there are operational or political constraints to quantitative easing, or fiscal debt is already at unstainable levels.

\section{Robustness}

We provide a number of robustness checks. In addition to reporting results based on an alternative FCI constructed using fixed parameters in Appendix B, we consider a corporate bond spread in place of the FCI, adding multiple lags of the explanatory variables, disaggregating nonfinancial credit, and for the US, incorporating monetary policy residuals. We also estimate the model for a sample that excludes the global financial crisis (GFC), and find that coefficients on FCI for the $5^{\text {th }}$ and $50^{\text {th }}$ percentile remain statistically different in the near-term, but the magnitude of the intertemporal risk tradeoff in the medium-term is reduced. But given that excluding the GFC removes the most negative growth outcomes and the GDP growth distribution is less negatively skewed, it is not surprising that the size of downside tail risks are smaller. 


\section{A. Alternative Financial Indicator and Credit Variables}

The results reported above are for a specific FCI constructed for each country based on Koop and Korobilis (2014). As discussed in section 3, we present estimates in Appendix B for an alternative construction of the FCI based on fixed parameters. We find they do not materially change the effects of financial conditions on the predicted distribution of GDP growth.

In addition, given the predicted negative effects of the excess bond premium and corporate bond spreads on expected growth (Gilchrist and Zakrajsek, 2012), we evaluate whether corporate bond spreads can help to predict the $5^{\text {th }}$ percentile of predicted GDP growth apart from their contribution to the FCI. We find that coefficients on corporate bond spreads for the $5^{\text {th }}$ percentile show the same pattern as coefficients on the FCI, which show that loose credit spreads are associated with a tighter left tail in the near-term, but a wider left tail in the medium-term (Figure 11a). However, coefficients on FCI are largely unchanged when corporate bond spreads are also included separately. These results suggest financial variables other than corporate bond spreads are relevant. ${ }^{21}$ While it would be possible to include simultaneously many separate financial indicators to distinguish possible effects, we would lose the efficiency of a parsimonious index and a single interaction term with growth in credit-to-GDP.

\footnotetext{
${ }^{21}$ We tried a similar exercise for equity return volatility and the change in the exchange rate, and while the credit boom variable based on equity return volatility for the $5^{\text {th }}$ percentile was significant, coefficients for median and $5^{\text {th }}$ percentile of output growth were not as significant as for corporate bond spreads, and FCI coefficients remained highly significant after these variables also were included. In addition, the results above for a financial firm EDF index, which also were significant, cannot be compared directly because there is more limited data and those estimations are based on a shorter time period, fewer countries, and a model without credit growth.
} 
Figure 11. Robustness Analysis

a. Corporate bond spread coefficients

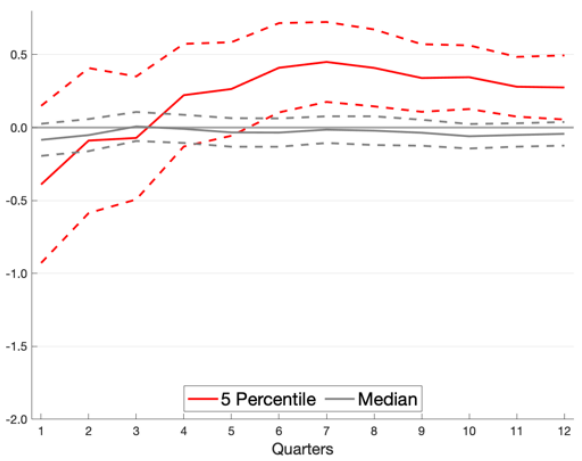

c. GaR by $\mathrm{FCl}$ groups, excluding GFC, credit boom

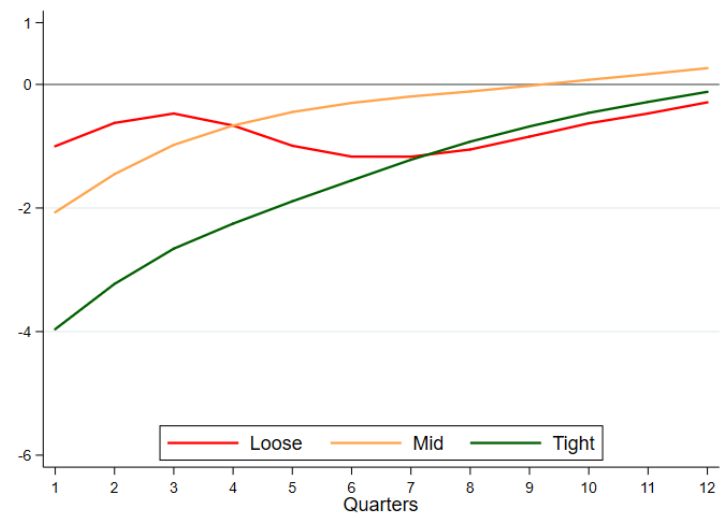

b. $\mathrm{FCl}$ coefficients on 5th percentile, US, Taylor rule

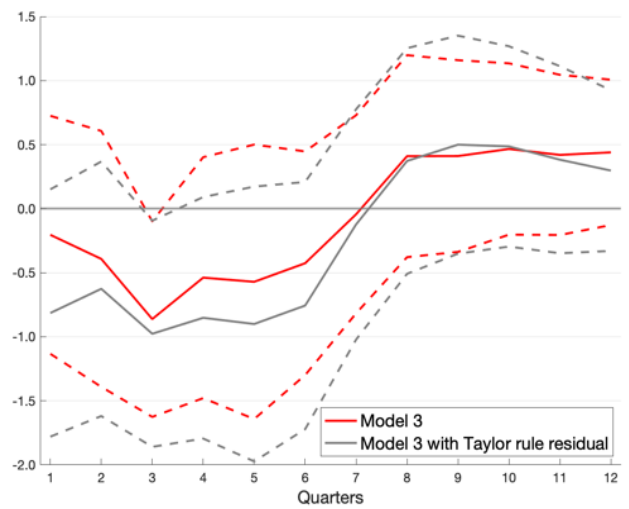

d. Difference of median and GaR, Loose - mid FCl, excluding GFC, credit boom

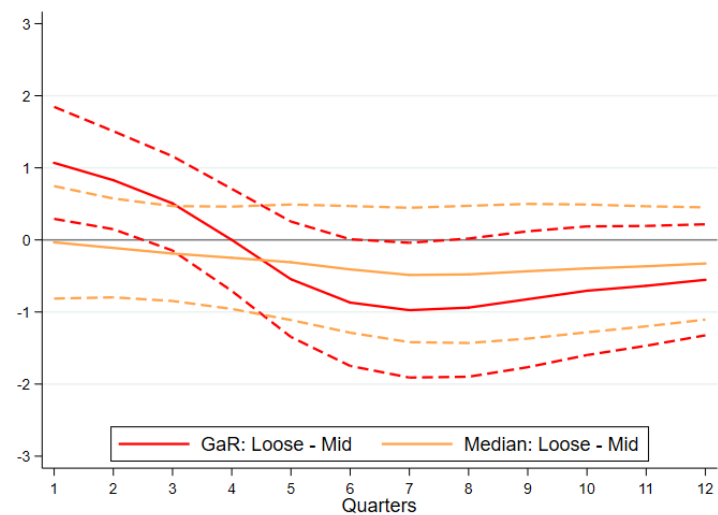

Source: IMF staff estimates.

Note: Panel a plots the coefficients on corporate bond spreads for the 5th percentile and median where spreads replace the $\mathrm{FCl}$ in model 1. Panel b plots coefficient estimates from model 1 for the US only on $\mathrm{FCl}$ and $\mathrm{FCl}$ adjusted for monetary policy from equations (B.1) and (B.2) with and without Taylor rule residuals. Bands are 90 percent confidence intervals. Panel c plots the GaR term structures excluding 2005-09, and defines credit boom by the interaction of loosest two deciles of $\mathrm{FCl}$ and highest two deciles of nonfinanical credit-to-GDP growth. The GaR estimates are grouped on initial tight, loose, and mid (middle four deciles) FCl levels. Panel d plots the difference in projected $\mathrm{GaR}$ and median between loose and mid $\mathrm{FCl}$ for the sample excluding 2005-09. Estimates in all panels are based on quantile regressions with local projection estimation methods, and standard errors are from bootstrapping techniques. Advanced economies include 11 countries with data for most from 1973 to 2017. Real GDP growth, vertical axis, is measured in percent. 
We also test for the possibility that the observed effects of FCI on GaR reflect monetary policy rather than the price of risk in a financial conditions index. Brunnermeier et al. (2017) emphasize that in the US, monetary policy affects GDP growth, and also risk spreads and credit, and it is important to separate the effects of financial variables on GDP growth from the effects of monetary policy. They focus on GDP growth but not the full distribution of future GDP growth. To incorporate monetary policy, we first re-estimate the FCI for the US to control for current monetary policy, in the same way the FCI controls for macroeconomic conditions, as shown in equations (B.1) and (B.2) in Appendix B. The re-estimation does not significantly change the adjusted FCI, likely because we already controlled for output growth and inflation in the estimation of the original FCI. We then add residuals of the policy rate from a Taylor rule specification to the quantile regressions for the US, while using the reestimated FCI. Residuals are based on the original specification of the Taylor rule from the St. Louis FRED database. ${ }^{22}$

The estimated coefficients on the adjusted FCI for the $5^{\text {th }}$ percentile of projected growth after adding the residuals are very similar to those without, and are positive at near-term horizons and switch to negative in the medium-term (Figure 11b). The reversal effects with the residuals are statistically different at 68 percent, though the reversal shown at 90 percent is less so, reflecting the model is estimated on only the US and does not take advantage of the panel. The corresponding GaR estimates from the model with the addition of the Taylor rule residuals differ only modestly from estimates without the residuals (not shown). These results for the US indicate the estimated effects of FCI on the GaR term structure cannot be attributed to not including monetary policy in the empirical model, though the addition of monetary policy affects the statistical significance of the reversal.

We also look at whether either business or household credit contributed disproportionately to the term structure of GaR. Some studies have found that household mortgage credit is an especially significant predictor of slow growth (Mian et al, 2015), and we can test whether it has a similarly important effect for the left tail of the growth distribution. For our panel of 11 countries, we do not find significant differences for the GaR term structure between the effects of household and business credit and, if anything, the effect of the credit boom is driven more by business credit than household credit (results not shown). This result may not be too surprising for our sample of advanced economies since the mid-1970s, which had many more business credit cycles than household real estate booms before the GFC.

\footnotetext{
22 https://fredblog.stlouisfed.org/2014/04/the-taylor-rule/. The results are basically unchanged when using an alternative Taylor rule which includes an interest rate smoothing parameter as in https://www.frbatlanta.org/cqer/research/taylor-rule.aspx?panel=1. Results shown with the Taylor rule residuals are based on estimations that exclude credit because some variables lose statistical significance with the larger set of explanatory variables when estimations are for a single country, the US, rather than the full panel of AEs.
} 
We also add lags of the independent variables to assess if the term structures may be reflecting delayed effects of financial conditions, GDP growth, or inflation. The addition of lags affects the coefficients on some of the independent variables measured at time $t$, but the resulting GaR estimates do not change materially. As shown in Appendix E, the term structure of GaR conditional on initial FCI groups based on a model with three lags for each independent variable show only very slight differences from those from model 3 without any lags.

\section{B. Quantile Estimates Excluding the Global Financial Crisis}

The GFC was a significant event, and the largest for many advanced economies since the Great Depression. We re-estimate model 3 excluding the five-year period, 2005 through 2009, a period that encompasses the financial crisis and recession, to evaluate the sensitivity to the GFC. We find that the coefficients on FCI remain negative and are statistically significant for the $5^{\text {th }}$ percentile of GDP growth in the near term and decline over the medium term. The coefficients on credit growth and credit boom also remain negative and significant, though the coefficients on credit boom are smaller than from estimates based on the full sample. These differences reflect GDP growth distributions with smaller left tails for most countries when the GFC period is excluded: For the 11 countries, seven had a higher $5^{\text {th }}$ percentile in the sample without the GFC period, three had the same value, and one had a lower $5^{\text {th }}$ percentile. In addition, there is less of a left skew in the real GDP growth distribution for the sample excluding the GFC (=-.272) than for the full sample $(=-.761)$.

The GaR term structures based on the sample excluding the GFC exhibit roughly the same shape as for the full sample, though the decline in GaR conditional on the loose decile of FCI over the forecast horizon is not as steep (Figure 11c). The differences in GaR for the loose FCI decile from the mid FCI deciles also are more modest than differences based on the full sample, but they still are positive and significant in the most near-term quarters and negative and significant for a portion of the medium-term (Figure 11d).

While it is useful to evaluate the sensitivity of results to dramatic episodes, it significantly shrinks the sample of left-tail experiences, which is precisely what we are trying to capture. Moreover, such episodes may not actually be extreme - the recent COVID-19 pandemic is an illustration of emerging large downside risks to GDP growth, albeit primarily from health risks than from the financial sector. In this case, financial conditions tightened as financial markets were disrupted, and could lead to higher downside risks to growth in the near-term where initial financial conditions were looser, and in the medium-term if credit boom conditions also were present. Over time, as the effects of the pandemic on real GDP growth are realized, our model could be used to assess out-of-sample accuracy of this tail event. 
The numerous robustness checks affirm strong empirical evidence for the significance of initial financial conditions and credit growth for future downside risks to GDP growth. It is also helpful to compare our paper to Plagborg-Moller et al. (2020) that offers a negative assessment of the value of financial conditions for forecasting GDP growth. A critical distinction of our paper from Plagborg-Moller et al. is that they are interested mainly in the marginal forecasting power of financial variables beyond information in a global factor which is already based on financial variables. Specifically, Plagborg-Moller et al. construct a global factor as a common factor from a macroeconomic data set with financial, real, monetary, and price variables, and then construct a separate financial factor that is orthogonal to the global factor to test for the additional forecasting power of financial variables. This approach reflects their primary focus on the value of financial variables only if they can better predict negative shocks and anticipate the timing of a recession than nonfinancial data. In contrast, our financial conditions index is more aligned with much of the research on financial conditions for economic activity which allows for contemporaneous interactions between real and financial variables (see comment by Gertler (2020), who emphasizes this point "their forecasting exercise is silent on the importance of financial conditions for economic activity.")

Another important distinction is that the global factor and orthogonal financial factor in Plagborg-Moller et al. combine financial price variables and credit quantity variables. But this is problematic because price and quantity variables differ in their cyclical behavior, which they acknowledge (see comment by Liang (2020)). Krishnamurthy and Muir (2020) show for a panel of countries from 1869 to 2008 that credit booms emerge earlier than spikes in corporate credit spreads ahead of a crisis (highlighted by Gertler (2020)). In our paper, we separate prices from credit and indeed show the effects of credit are stronger at the mediumterm horizon than at the very near-term. We report in table 3 that the effect of initial financial conditions on the lower left tail of GDP growth are significant in the near term and its effects change over the forecast period, while the effects of credit have their maximum effects at the medium-term horizon.

\section{Conclusion}

Since the global financial crisis and consequent damage to economic growth, more research has turned to exploring linkages between the financial sector and real economic activity. In this paper, we explore the empirical relationship between financial conditions and the distribution of real GDP growth for 11 AEs from 1975 to 2017. The relationships we examine are rooted in macrofinancial linkages arising from financial frictions, such as asymmetric information and regulatory constraints, where a low price of risk can lead to buildups of financial vulnerabilities which then can generate negative spillovers and contagion when the price of risk reverses. We employ a model of output growth that depends on financial conditions, credit growth, economic conditions, and inflation, using panel quantile regressions and local projections. This method generates the term structure for the 
distribution of forecasted growth, and we focus on the lower $5^{\text {th }}$ percentile of forecasted growth for horizons out to twelve quarters, which measures the term structure of growth-atrisk.

The main contributions of this paper are to show empirically that financial conditions affect the distribution of predicted GDP growth, and its effects differ over the projection horizon in a way that is consistent with an intertemporal risk tradeoff. Of course, there are many studies that have linked financial conditions to growth - indeed, many argue that monetary policy affects the economy through financial conditions. But we show based on panel estimates for 11 AEs that financial conditions affect the lower tail, not just the mean, of the predicted distribution, and that the signs of the coefficients on financial conditions reverse from the short to medium term horizons for the lower tail. Combined, the conditional projected growth distribution shifts with changes in financial conditions, with the lower tail, GaR, more responsive than the median or upper tail to financial conditions. Results using a GIV method suggest these results are causal and not simply due to endogeneity bias.

Of particular significance, looser initial financial conditions imply higher GaR (smaller downside risk) in the near-term, but these effects reverse and imply a lower GaR (greater downside risk) in the medium-term relative to outcomes from mid-range financial conditions. Moreover, the additional boost to predicted mean growth from initial loose financial conditions and high credit diminishes over the projection horizon, suggesting that expected growth has not increased to offset the costs of greater downside risks.

This empirical tradeoff is relevant to both macroeconomic forecasting and policymaking. The strong inverse correlation between conditional growth and conditional downside risk that we document is usually ignored in dynamic macroeconomic models, which assume often for computation reasons that growth is not affected by volatility, and vice versa (certainty equivalence). This omission can lead to an underestimate of downside risks in the future from loose initial financial conditions.

The GaR measure that we develop offers promise as a way to translate financial stability risks to macroeconomic performance. While progress has been made to add macrofinancial linkages, a dominant paradigm has not yet emerged about how to incorporate them into expanded models that could be used regularly by policymakers. Our empirical model takes a step forward to integration. The GaR measure ultimately could help in developing macroprudential policies. It can provide an objective gauge for downside risks to forecasted growth and thus whether macroprudential policy interventions are needed, as well as a metric of whether interventions have been successful. For example, it could be used to help calibrate the severity of stress test scenarios, a countercyclical capital buffer, or borrower loan-tovalue or loan-to-income ratios to build the resilience of the financial system. While structural models are needed for policy evaluation, our measures offer important data calibrations to fit. 
In addition, by expressing financial stability risks in terms of risks to output, they have the potential to be better incorporated into monetary policy decision making. When financial stability risks are expressed as the probability of a banking crisis, the discussion features discontinuous transitions of states, which sets up decision-making frameworks that consider the distribution of growth only intermittently. In our view, estimating the interplay of financial conditions and the conditional distribution in a continuous fashion has the advantage that it could become more relevant to policy making on a regular basis. Being able to express risks arising from the financial sector in the same terms as used in models for other macroeconomic policies will help when evaluating alternative policy options and foster more effective consultation and coordination. 


\section{References}

Adrian, Tobias, Nina Boyarchenko, and Domenico Giannone (2019) "Vulnerable Growth" American Economic Review, 109(4) April, pp. 1263-1289.

Adrian, Tobias, and Fernando Duarte (2018) "Financial Vulnerability and Monetary Policy" Federal Reserve Bank of New York Staff Reports 804.

Adrian, Tobias, Fernando Duarte, Federico Grinberg, Tommaso Mancini-Griffoli (2017) "Monetary Policy and Financial Conditions: A Cross-Country Study" In: Advancing the Frontiers of Monetary Policy, International Monetary Fund, edited by Adrian, Laxton, Obstfeld, pp. 83-106.

Adrian, Tobias, Fernando Duarte, Nellie Liang, and Pawel Zabcyzk (2019). "Monetary and Macroprudential Policy with Endogenous Risk," working paper.

Adrian, Tobias, Fernando Duarte, Nellie Liang, and Pawel Zabczyk (2020). "NKV: A New Keynesian Model with Vulnerability." AEA Papers and Proceedings, 110: 470-76.

Adrian, Tobias, and Nellie Liang (2018), "Monetary Policy, Financial Conditions, and Financial Stability," International Journal of Central Banking 14(1), pp. 73-131.

Adrian, Tobias, Erkko Etula, and Tyler Muir (2014). "Financial Intermediaries and the Cross-Section of Asset Returns," Journal of Finance 69(6), pp. 2557-2596

Adrian, Tobias, and Hyun Song Shin (2014). "Procyclical Leverage and Value-at-Risk," Review of Financial Studies 27(2), 373-403.

Aikman, David, Andreas Lehnert, Nellie Liang, and Michele Modugno (2020). "Credit, Financial Conditions, and Monetary Policy Transmission," International Journal of Central Banking 16(3), 140-179.

Aldasoro, I., Beltran, P., Grinberg, F., \& Mancini-Griffoli, T. (2019), International Bank Lending and EMEs. A Granular Instrumental Variable Approach. Forthcoming IMF Working Paper.

Bernanke, Ben, Mark Gertler, "Agency Costs, Net Worth, and Business Fluctuations," American Economic Review, 79(1), 14-31.

Bernanke, Ben, Mark Gertler, and Simon Gilchrist (1999), "The Financial Accelerator in a Quantitative Business Cycle Framework," Handbook of Macroeconomics, J.B. Taylor and M. Woodford (ed.), Elsevier, Chapter 21, 1341-1393.

Borio, Claudio and Philip Lowe (2002). "Asset Prices, Financial and Monetary Stability: Exploring the Nexus," BIS Working Paper 114. 
Brunnermeier, Markus K., Darius Palia, Karthik Sastry, and Christopher Sims (2017).

"Feedbacks: Financial Markets and Economic Activity," Working paper, Dec.

Brunnermeier, Markus K., and Lasse Heje Pedersen (2009). "Market Liquidity and Funding Liquidity," Review of Financial Studies, 22(6), 2201-2238.

Brunnermeier, Markus K., and Yuliy Sannikov (2014a) "A Macroeconomic Model with a Financial Sector” American Economic Review 104(2), 379-421.

Brunnermeier, Markus K., and Yuliy Sannikov (2014b). "Monetary Analysis: Price and Financial Stability," Paper presented at the ECB Forum on Central Banking, 26 May 2014, Sintra, Portugal.

Canay, Ivan A. (2011). "A Simple Approach to Quantile Regression for Panel Data," The Econometrics Journal, 14(3), 368-386.

Cech, Frantisek and Jozef Barunik (2017). "Measurement of Common Risk Factors: A Panel Quantile Regression Model for Returns," Institute of Economic Studies, Charles University, Working Paper, 20

Cerra, Valerie and Sweta Chaman Saxena (2008). "Growth Dynamics: The Myth of Economic Recovery,” American Economic Review, 98:1, 439-457.

Chavleishvili, S. and S. Manganelli (2019), "Forecasting and Stress Testing with Quantile Vector Autoregression," ECB Working Paper No. 2330.

Doz, Catherine, Domenico Giannone, and Lucrezia Reichlin (2011). "A two-step estimator for large approximate dynamic factor models based on Kalman filtering," Journal of Econometrics, 164(1), 188-205.

Duprey, Thibaut, and Alexander Ueberfeldt (2020), “Managing GDP Tail Risk,” No. 20-3. Bank of Canada.

Freedman, D.A. (1981). "Bootstrapping Regression Models," Annals of Statistics, 9, 12181228.

Gabaix, Xavier and Ralph Koijen, R. S. (2019). Granular Instrumental Variables. Available at SSRN 3368612.

Galvao, Antonio F., and Gabriel Montes-Rojas (2015). “On Bootstrap Inference for Quantile Regression Panel Data: A Monte Carlo Study,” Econometrics, 3, 645-666.

Giglio, Stefano, Bryan Kelly, and Seth Pruitt (2016), "Systemic Risk and the Macroeconomy: An Empirical Evaluation," Journal of Financial Economics 119, 457-471. 
Gilchrist, Simon, and Egon Zakrajšek (2012). "Credit Spreads and Business Cycle Fluctuations," American Economic Review 102(4), 1692-1720.

Greenwood, Robin and Samuel Hanson (2013). "Issuer Quality and Corporate Bond Returns,” The Review of Financial Studies 26(6), 1483-1525.

Gennaioli, Nicola, and Andrei Shleifer. A Crisis of Beliefs: Investor Psychology and Financial Fragility. Princeton University Press, 2018.

Gertler, Mark (2020), “Discussion of: When is Growth at Risk?” Brookings Papers on Economic Activity Spring 2020, June (Forthcoming).

He, Zhiguo, and Arvind Krishnamurthy (2013). "Intermediary Asset Pricing," American Economic Review 103(2), 1-42.

He, Zhiguo, and Arvind Krishnamurthy (2012). "A Macroeconomic Framework for Quantifying Systemic Risk," SSRN Electronic Journal, November 2012, DOI:

10.2139/ssrn.2133412.

Jordà, Òscar (2005). "Estimation and Inference of Impulse Responses by Local Projections," American Economic Review, Vol. 95, No. 1, pp.161-182.

Jordà, Òscar, Moritz Schularick, and Alan M. Taylor (2013) "When Credit Bites Back" Journal of Money, Credit and Banking 45(2) pp. 3-28.

International Monetary Fund (2017), Global Financial Stability Report, Chapter 3, "Growth at Risk: A Macroeconomic Measure of Financial Stability," Oct.

Kapetanios, George (2008). "A Bootstrap Procedure for Panel Datasets with Many CrossSectional Units," Econometrics Journal, 11, 377-395.

Kiley, Michael T. (2018). "Unemployment Risk," Finance and Economics Discussion Series, Board of Governors of the Federal Reserve System 2018-67.

Koenker, Roger (2004). “Quantile Regression for Longitudinal Data.” Journal of Multivariate Analysis, 91, 74-89.

Koop, Gary, and Dimitris Korobilis (2014). "A new index of financial conditions," European Economic Review, 71, 101-116.

Korinek, Anton, and Alp Simsek (2016). "Liquidity trap and excessive leverage," American Economic Review 106(3), pp. 699-738.

Krishnamurthy, Arvind, and Tyler Muir (2020). "How Credit Cycles Across a Financial Crisis," National Bureau of Economic Research w23850, September 2017, revised September 2020. 
Lahiri, S. N. (2003). "Resampling Methods for Dependent Data," New York: Springer.

Lamarche, Carlos. (2010). "Robust Penalized Quantile Regression Estimation for Panel Data," Journal of Econometrics, 157(2), 396-408.

Liang, J. Nellie (2020). "Discussion of: When is Growth at Risk?" Brookings Papers on Economic Activity Spring 2020, June (Forthcoming).

López-Salido, David, Jeremy C. Stein, and Egon Zakrajšek (2017). “Credit-market Sentiment and the Business Cycle," Quarterly Journal of Economics 132(3), pp.1373-1426

Mian, Atif, Amir Sufi, and Emil Verner (2015) "Household Debt and Business Cycles Worldwide," National Bureau of Economic Research 21581.

Minsky, Hyman P. (1986). "Stabilizing an Unstable Economy”. New Haven: Yale University Press.

Plagborg-Møller, Mikkel, Lucrezia Reichlin, Giovanni Ricco, and Thomas Hasenzagl (2020). "When is Growth at Risk?" Brookings Papers on Economic Activity Spring 2020, revised May 4, (Forthcoming).

Primiceri, Giorgio E. (2005). "Time varying structural vector autoregressions and monetary policy,” The Review of Economic Studies, 72(3), 821-852.

Ramey, Valerie A. (2016). "Macroeconomic shocks and their propagation," In Handbook of Macroeconomics (Vol. 2, pp. 71-162). Elsevier.

Stock, James H. and Mark W. Watson, "Identification and Estimation of Dynamic Causal Effects in Macroeconomics", NBER working paper 24216 (2018)

Wolfers, Justin (2003), "Is Business Cycle Volatility Costly? Evidence from Surveys of Subjective well-being." International Finance, Spring, 6 (1), 1-26. 


\section{Appendix A. Start Dates for Model Estimation and for Individual Components of FCI}

\begin{tabular}{|c|c|c|c|c|c|c|}
\hline Country & $\begin{array}{c}\text { Start date for } \\
\text { estimation }\end{array}$ & Interbank Spread & Corporate Spread & $\begin{array}{l}\text { Sovereign } \\
\text { Spread }\end{array}$ & Term Spread & $\begin{array}{c}\text { Equity Returns } \\
\text { Volatility }\end{array}$ \\
\hline AUS & $1975 q 1$ & $1979 q 1$ & 1983q2 & $1973 q 1$ & $1979 q 1$ & $1973 q 1$ \\
\hline CAN & 1981q2 & $1973 q 1$ & $1979 q 1$ & $1973 q 1$ & $1973 q 1$ & $1973 q 1$ \\
\hline CHE & $1980 q 2$ & $1980 q 1$ & $1982 q 1$ & $1979 q 1$ & $1980 q 1$ & $1973 q 1$ \\
\hline DEU & $1975 q 1$ & $1979 q 1$ & $1977 q 1$ & $1977 q 1$ & $1979 q 1$ & $1973 q 1$ \\
\hline ESP & $1992 q 1$ & $1990 q 1$ & $1990 q 1$ & $1990 q 1$ & $1990 q 1$ & $1990 q 1$ \\
\hline FRA & $1980 q 3$ & $1979 q 1$ & $1979 q 1$ & $1977 q 1$ & $1979 q 1$ & $1973 q 1$ \\
\hline GBR & $1975 q 1$ & $1973 q 1$ & $1979 q 1$ & $1973 q 1$ & $1973 q 1$ & $1973 q 1$ \\
\hline ITA & 1981q2 & $1979 q 1$ & $1979 q 1$ & $1977 q 1$ & $1979 q 1$ & $1973 q 1$ \\
\hline JPN & $1975 q 3$ & $1979 q 1$ & $1973 q 1$ & $1973 q 1$ & $1979 q 1$ & $1973 q 1$ \\
\hline SWE & $1980 q 2$ & $1979 q 1$ & $1979 q 1$ & $1979 q 1$ & $1979 q 1$ & $1973 q 1$ \\
\hline USA & $1975 q 1$ & $1973 q 1$ & $1973 q 1$ & $1973 q 1$ & $1973 q 1$ & $1973 q 1$ \\
\hline
\end{tabular}

\begin{tabular}{|c|c|c|c|c|c|c|}
\hline Country & Equity Returns & $\begin{array}{l}\text { Change in real } \\
\text { long - term rate }\end{array}$ & Change in FX & VIX & MOVE & $\begin{array}{l}\text { House price } \\
\text { return }\end{array}$ \\
\hline AUS & $1973 q 1$ & $1973 q 1$ & 1970q1 & $1986 q 1$ & $1988 q 2$ & $1973 q 1$ \\
\hline CAN & $1973 q 1$ & $1973 q 1$ & $1970 q 1$ & $1986 q 1$ & $1988 q 2$ & $1973 q 1$ \\
\hline CHE & $1973 q 1$ & $1979 q 1$ & $1970 q 1$ & $1986 q 1$ & $1988 q 2$ & $1973 q 1$ \\
\hline DEU & $1973 q 1$ & $1977 q 1$ & $1971 q 1$ & $1986 q 1$ & $1988 q 2$ & $1973 q 1$ \\
\hline ESP & $1990 q 1$ & $1992 q 1$ & $1971 q 1$ & $1986 q 1$ & $1988 q 2$ & $1990 q 2$ \\
\hline FRA & $1973 q 1$ & $1973 q 1$ & $1971 q 1$ & $1986 q 1$ & $1988 q 2$ & $1973 q 1$ \\
\hline GBR & $1973 q 1$ & $1973 q 1$ & $1970 \mathrm{q} 1$ & $1986 q 1$ & $1988 q 2$ & $1973 q 1$ \\
\hline ITA & $1973 q 1$ & $1973 q 1$ & $1971 q^{1}$ & $1986 q 1$ & $1988 q 2$ & $1973 q 1$ \\
\hline JPN & $1973 q 1$ & $1973 q 1$ & $1970 \mathrm{q} 1$ & $1986 q 1$ & $1988 q 2$ & $1973 q 1$ \\
\hline SWE & $1973 q 1$ & $1979 q 1$ & 1970q1 & $1986 q 1$ & $1988 q 2$ & $1973 q 1$ \\
\hline USA & $1973 q 1$ & $1973 q 1$ & $1970 q 1$ & $1986 q 1$ & $1988 q 2$ & $1973 q 1$ \\
\hline
\end{tabular}

\begin{tabular}{|c|c|c|c|c|c|c|}
\hline Country & $\begin{array}{c}\text { Change in equity } \\
\text { market } \\
\text { capitalization of } \\
\text { financial sector to } \\
\text { total market }\end{array}$ & $\begin{array}{c}\text { Domestic } \\
\text { commodity price } \\
\text { inflation }\end{array}$ & $\begin{array}{l}\text { Equity trading } \\
\text { volume }\end{array}$ & $\begin{array}{c}\text { Market } \\
\text { capitalization for } \\
\text { equities }\end{array}$ & $\begin{array}{c}\text { Market } \\
\text { capitalization for } \\
\text { bonds }\end{array}$ & $\begin{array}{c}\text { Expected default } \\
\text { frequencies for } \\
\text { banks }\end{array}$ \\
\hline AUS & $2000 q 1$ & $1970 q 1$ & $1994 q 2$ & $2001 q 1$ & $1995 q 4$ & $1999 q 4$ \\
\hline CAN & $2000 q 1$ & $1970 q 1$ & $1990 q 4$ & $2000 q 3$ & $1995 q 4$ & $1999 q 4$ \\
\hline CHE & $2000 q 1$ & $1970 q 1$ & $1994 q 2$ & $2002 q 4$ & $1995 q 4$ & $1999 q 4$ \\
\hline DEU & $2000 q 1$ & $1970 q 1$ & $1993 q 4$ & $1973 q 4$ & $1995 q 4$ & $1999 q 4$ \\
\hline ESP & $2000 q 2$ & 1970q1 & $1992 q 4$ & $2001 q 2$ & $1995 q 4$ & $1999 q 4$ \\
\hline FRA & $2000 q 1$ & $1970 q 1$ & $1993 q 4$ & $1988 q 4$ & $1995 q 4$ & $1999 q 4$ \\
\hline GBR & $2000 \mathrm{q} 1$ & $1970 \mathrm{q} 1$ & $1993 q 4$ & $1986 q 4$ & $1995 q 4$ & $1999 q 4$ \\
\hline ITA & $2016 q 1$ & 1970q1 & $2004 q 2$ & $2004 q 2$ & $1995 q 4$ & $1999 q 4$ \\
\hline JPN & $2000 q 1$ & $1970 q 1$ & $1993 q 4$ & $1989 q 4$ & $1995 q 4$ & $1999 q 4$ \\
\hline SWE & $2000 q 1$ & $1970 q 1$ & $1993 q 4$ & $2001 q 2$ & $1995 q 4$ & $1999 q 4$ \\
\hline USA & $2000 q 1$ & $1970 q 1$ & $1990 q 4$ & $2001 q 2$ & $1995 q 4$ & $1999 q 4$ \\
\hline
\end{tabular}




\section{Appendix B. Constructing FCI and Sensitivity Analysis}

The model for TVP-FAVAR model to estimate the FCI takes the following form:

(B.2) $\left[\begin{array}{l}Y_{t} \\ f_{t}\end{array}\right]=c_{t}+B_{t, 1}\left[\begin{array}{l}Y_{t-1} \\ f_{t-1}\end{array}\right]+\cdots+B_{t, p}\left[\begin{array}{l}Y_{t-p} \\ f_{t-p}\end{array}\right]+\varepsilon_{t}$

In (B.1), $Z_{t}$ is an observed vector of financial variables, $Y_{t}$ is a vector of macroeconomic variables of interest (in our application, real GDP growth and CPI inflation), $\theta_{t}^{y}$ are regression coefficients, $\theta_{t}^{f}$ are the factor loadings, and $f_{t}$ is the latent factor, interpreted as the FCI. The time-varying coefficients and the latent factor are unobserved. If coefficients were to be restricted to be constant, the model would collapse to a factor augmented vector autoregressive (FP-FAVAR) model as proposed by Bernanke et al. (2005). Equation (B.1) allows us to extract the latent FCI from financial variables $Z_{t}$. In equation (B.2), $B_{t, 1} \ldots B_{t, p}$ are time-varying coefficients, which allows us to model the dynamic interactions of the FCI with macroeconomic variables $Y_{t}$. The factor $f_{t}$ is estimated using $\theta_{t}, B_{t}$ using the Kalman filter (using observables up to $t$ ) and smoother (using estimated data up to $t+1$ conditional on observables up to $\mathrm{t}$ ). ${ }^{23}$ Unlike univariate forecasting regressions, the model uses of a multivariate system as specified by (A.2) to forecast macroeconomic variables using the FCI. Thus, we jointly model all the variables in the system which should allow us to better characterize their co-movements and interdependence. Second, we are able to purge the FCI from the effect of current macroeconomic conditions. Thus, the estimated FCI reflects information solely associated with the financial sector. ${ }^{24}$ Relative to FP-FAVAR, the TVPFAVAR models allows for dynamic interactions between the FCIs and macroeconomic conditions which can evolve over time, and allows for differences in starting dates for some financial indicators with a flexible estimation procedure.

The model is completed by specifying vectors of loadings $\theta_{t}$ and $B_{t}$ that evolve as multivariate random walks with time-varying covariances. Identification is achieved in a standard fashion by restricting the time-varying covariance matrix to be diagonal. This restriction ensures that the factors capture movements that are common to the financial variables after removing the effect of current macroeconomic conditions.

\footnotetext{
${ }^{23}$ Note that this does not introduce look ahead bias in the estimation.

${ }^{24}$ We omit the model selection and averaging proposed by Koop and Korobilis (2014) as our focus is not to find the best possible FCI to forecast the conditional mean of macroeconomic conditions.
} 
The model is estimated using the algorithm in Koop and Korobilis (2014) that circumvents the need of slower Bayesian simulation algorithms that may be computationally more demanding. It combines variance discounting methods with the Kalman filter to obtain analytical results for the posteriors of the state variable $f_{t}$. as well as the time-varying parameters $\left(\theta_{t}, B_{t}\right)$. The dual linear Kalman filter is as follows: first update the parameters $\left(\theta_{t}, B_{t}\right)$ and subsequently update the factor $f_{t}$ given the parameter estimate. This conditioning allows to use two distinct linear Kalman filters or smoothers: one for the parameters and one for the unobserved factor. It is based on the Kalman filter and smoother and in the case of estimating models and can be sketched as follows (further details can be found in Koop and Korobilis (2014) Technical Appendix):

1. a. Initialize all parameters

b. Obtain the principal components estimates of the factors $f_{t}$.

2. Estimate the time varying parameters given $f_{t}$

a. Estimate covariance matrices using Variance Discounting

b. Estimate $\left(\theta_{t}, B_{t}\right)$ given the covariance matrices using the Kalman filter (using data up to $t$ ) and smoother (using data up to $t+1$ )

3. Estimate the factor $f_{t}$ using $\left(\theta_{\mathrm{t}}, \mathrm{B}_{\mathrm{t}}\right)$ using the Kalman filter (using data up to $t$ ) and smoother (using estimated data up to $t+1$ ).

For comparison, Panel B1 shows the time series of the FCI estimated using the TVP-FAVAR model and a FP-FAVAR model that restricts parameters to be constant over time. To overcome the unbalanced data availability and maintain the same sample coverage, missing values are replaced by zeros as usual practice when estimating factor models. It is apparent that the series are very similar, with a lowest correlation for Germany of 0.9. We use the FPFAVAR model for the FCI (called PCA) to re-estimate our baseline model presented in equations A.1 and A.2 The results are presented in Table B1 and are similar to results from model based on FCI with TVP-FAVAR (and presented in Table 2 of the main text of this paper). 


\section{Panel B1: Comparison of FCls Constructed with TVP-FAVAR and FP-FAVAR by Country}
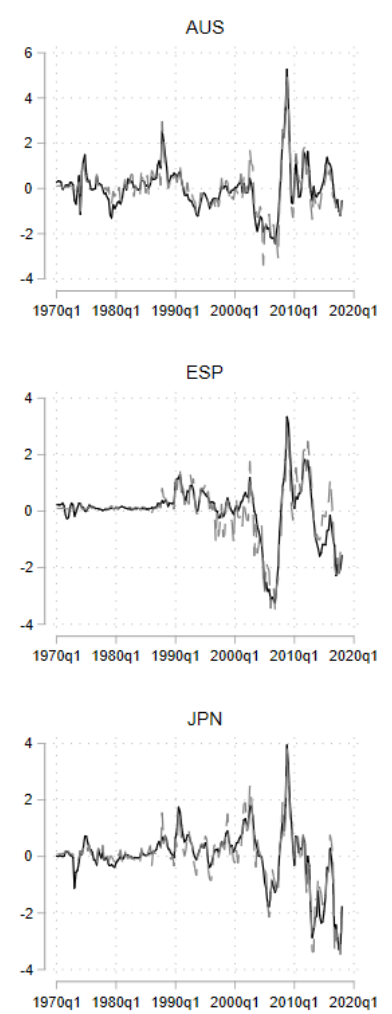
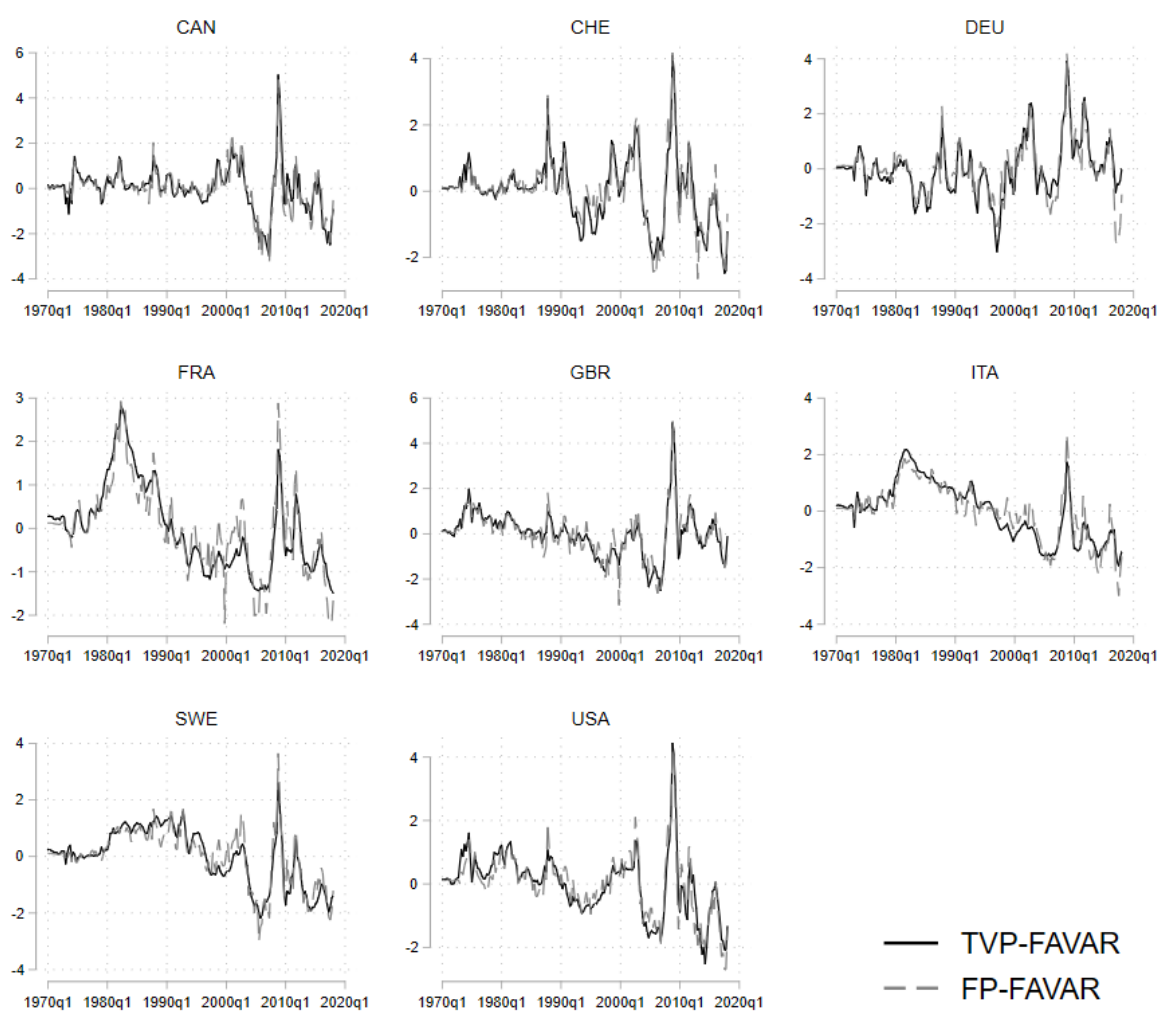

Note: This panel compares different $\mathrm{FCl}$ estimations. TVP-FAVAR is the time-varying parameter VAR follows the specification from equations (B.1) and (B.2). FP-FAVAR imposes that the parameters are fixed over time. To balance data availability and allow for estimation over the same period, missing observations are replaced with zeros in FP-FAVAR.

\section{Table B1: Estimated Coefficients for the $5^{\text {th }}$ Percentile with $\mathrm{FCl}$ Alternative Based on} FP-FAVAR (PCA)

\begin{tabular}{|l|llllll|}
\hline \multicolumn{7}{|l|}{ Model 3: PCA FCI, GDP growth, Inflation, Credit growth and Credit boom } \\
\hline & $\mathrm{h}=2$ & $\mathrm{~h}=4$ & $\mathrm{~h}=6$ & $\mathrm{~h}=8$ & $\mathrm{~h}=10$ & $\mathrm{~h}=12$ \\
\hline PCA & $-2.383^{* * *}$ & $-2.321^{* * *}$ & $-1.482^{* * *}$ & -0.290 & 0.169 & $0.363^{* *}$ \\
& $(0.535)$ & $(0.589)$ & $(0.445)$ & $(0.371)$ & $(0.240)$ & $(0.171)$ \\
GDP growth & $0.270^{* * *}$ & $0.190^{* * *}$ & $0.136^{* * *}$ & 0.060 & 0.023 & 0.018 \\
& $(0.073)$ & $(0.049)$ & $(0.050)$ & $(0.038)$ & $(0.029)$ & $(0.025)$ \\
Inflation rate & $-0.332^{* * *}$ & $-0.258^{* * *}$ & $-0.231^{* * *}$ & $-0.154^{* *}$ & $-0.089^{*}$ & -0.056 \\
& $(0.091)$ & $(0.082)$ & $(0.083)$ & $(0.065)$ & $(0.047)$ & $(0.038)$ \\
Credit-to-GDP growth & $-0.291^{*}$ & $-0.401^{* *}$ & $-0.438^{* *}$ & $-0.384^{* *}$ & $-0.427^{* * *}$ & $-0.418^{* * *}$ \\
& $(0.171)$ & $(0.161)$ & $(0.175)$ & $(0.179)$ & $(0.125)$ & $(0.103)$ \\
Credit boom & 0.060 & -0.414 & $-2.313^{* * *}$ & $-2.136^{* * *}$ & $-1.121^{* * *}$ & -0.407 \\
& $(0.436)$ & $(0.478)$ & $(0.697)$ & $(0.544)$ & $(0.410)$ & $(0.317)$ \\
\hline
\end{tabular}

Note: This table reports coefficients and standard errors for model 3 using the $\mathrm{FCl}$ generated by the FP-FAVAR model given by equations (B.1) and (B.2). 


\section{Appendix C: FCI and Credit-to-GDP Growth}
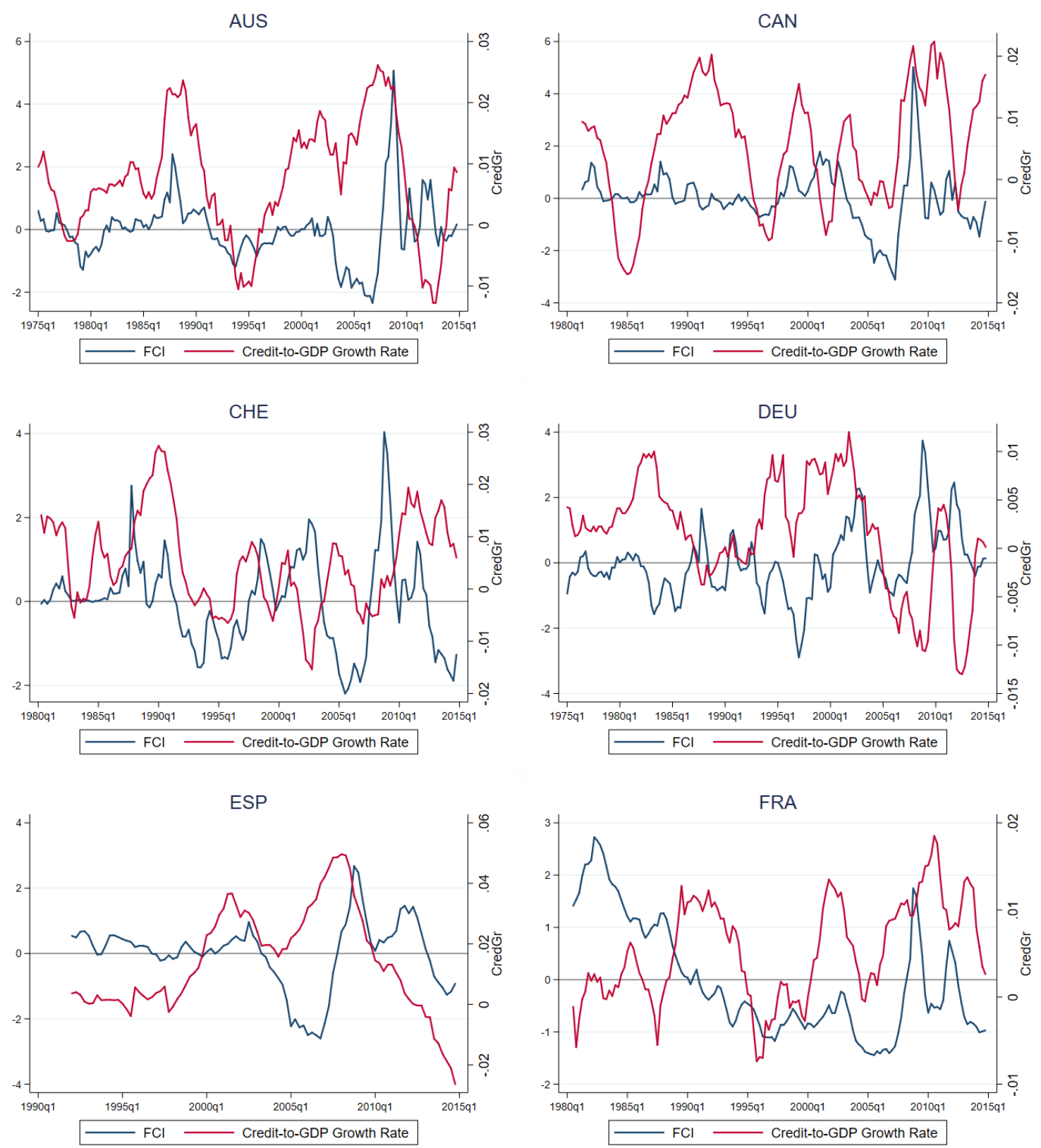
GBR

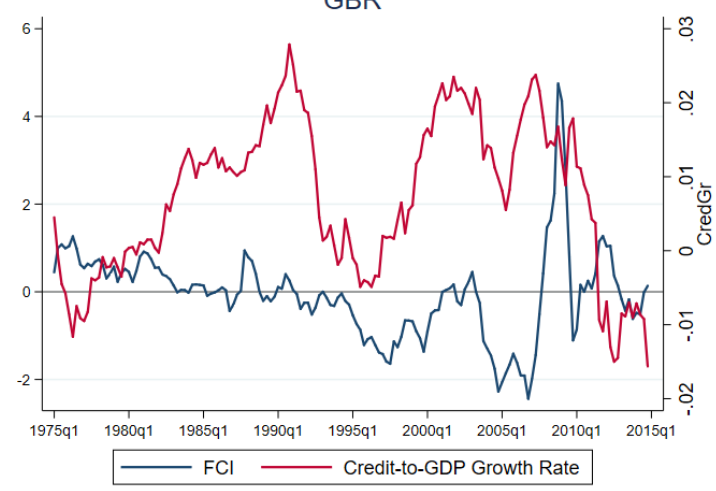

JPN

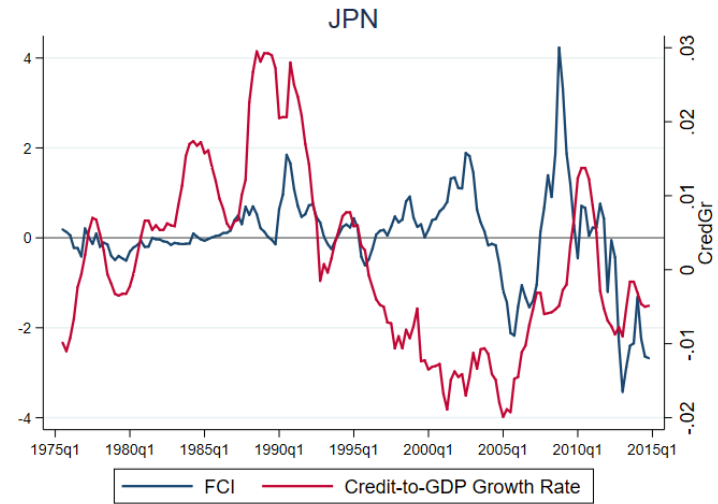

USA

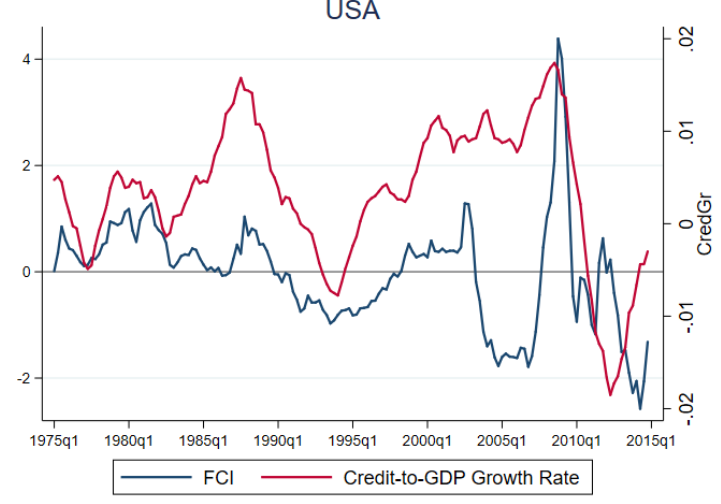

ITA

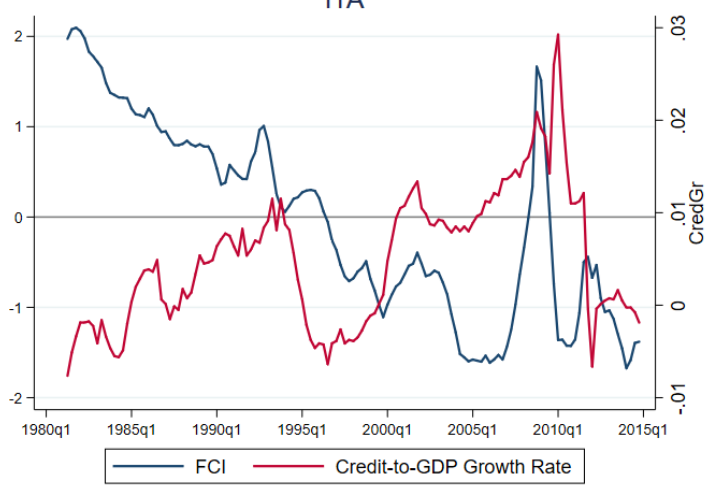

SWE

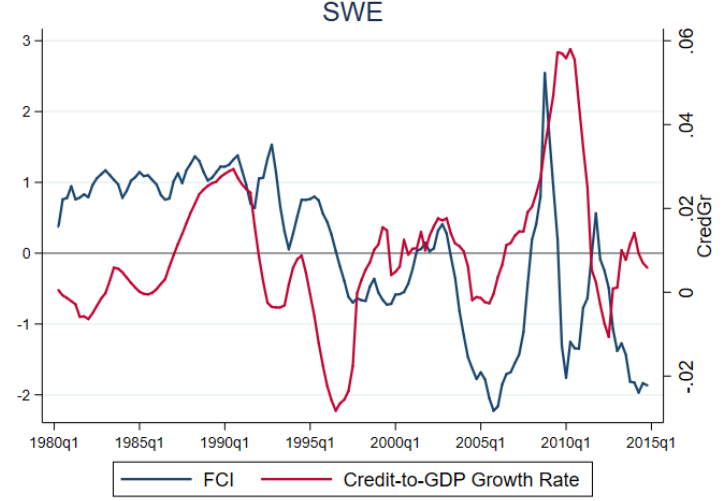




\section{Appendix D: Probability Density Functions from Models 1 and 2, by FCI Groups and Projection Horizons}

Model $1, h=4$

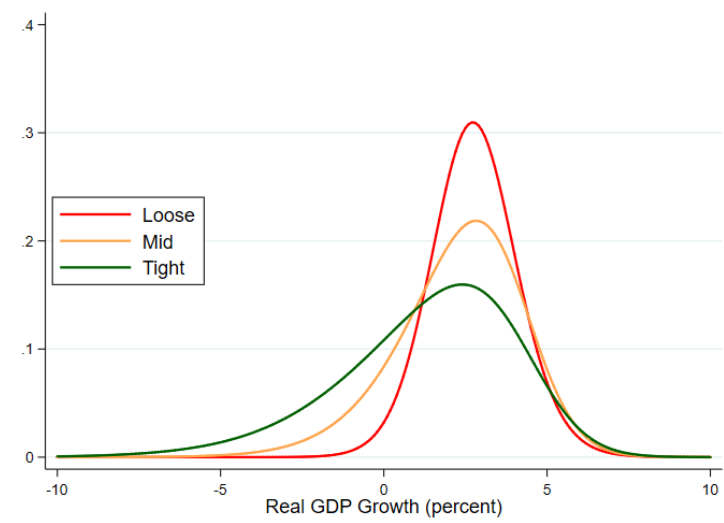

Model $2, \mathrm{~h}=4$

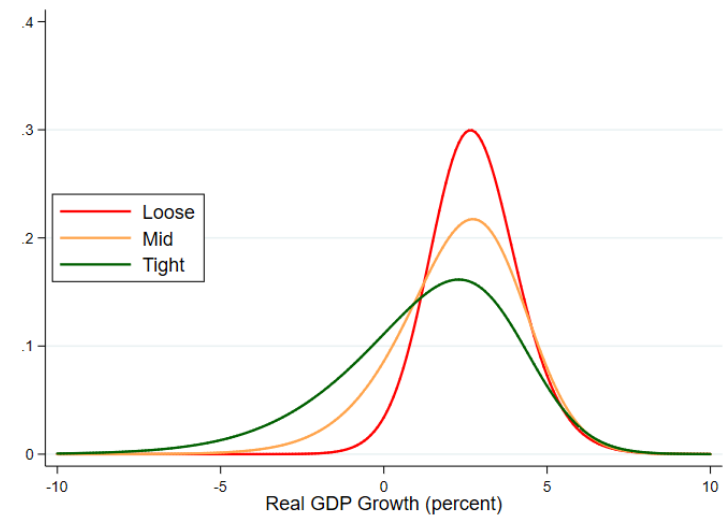

Model $1, h=12$

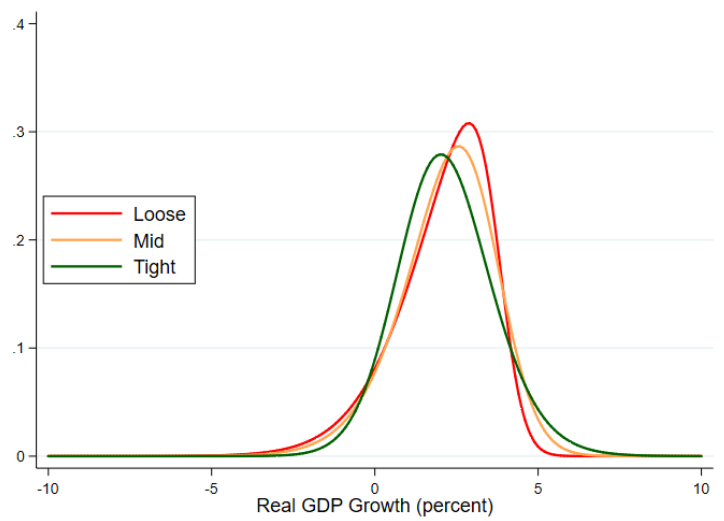

Model $2, \mathrm{~h}=12$

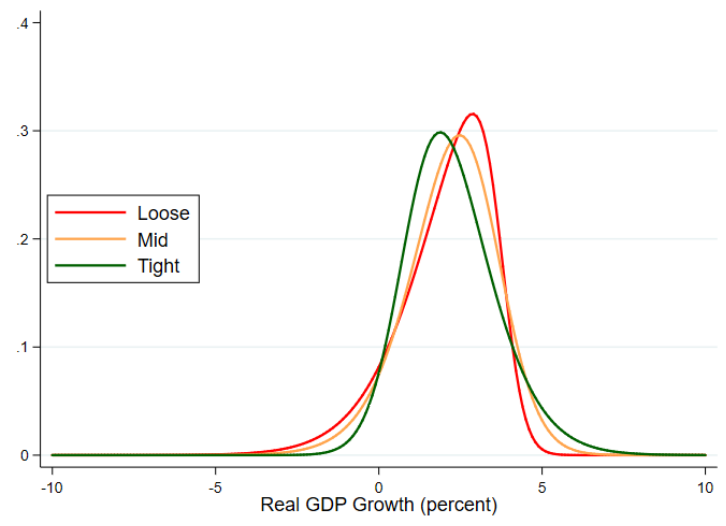

Note: Probability density functions shown are estimated based on models 1 and 2 using panel quantile regression methods and local projections and fitted to a skewed t distribution. They are shown for the loosest decile, the tightest decile, and mid (middle four deciles) of $\mathrm{FCl}$, for $\mathrm{h}=4$ and $\mathrm{h}=12$ quarters. Real GDP growth, horizontal axis, is measured in percent. Advanced economies include 11 countries most with data from 1973 to 2017. 


\section{Appendix E: Term Structure of GaR, Model 3, No Lags and Three Lags}

a. Model 3, no lags, Credit Boom

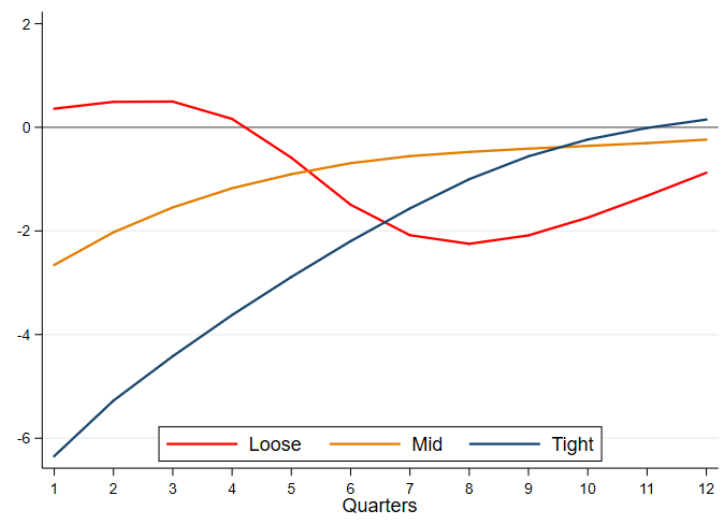

c. Model 3, no lags, Not Boom

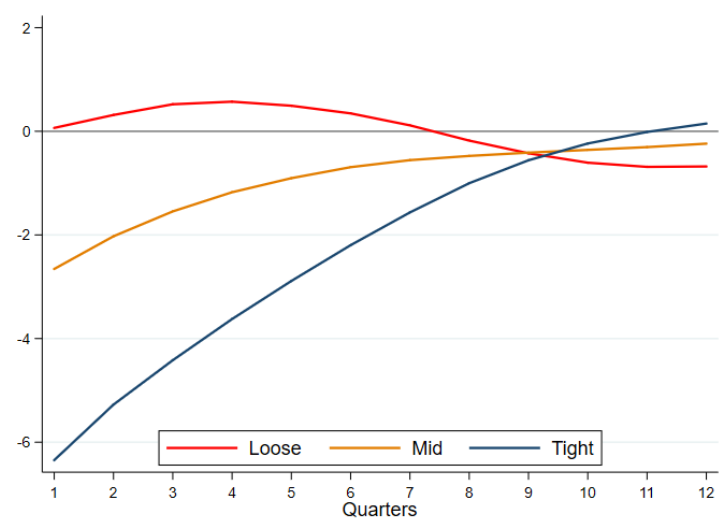

b. Model 3, three lags, Credit Boom

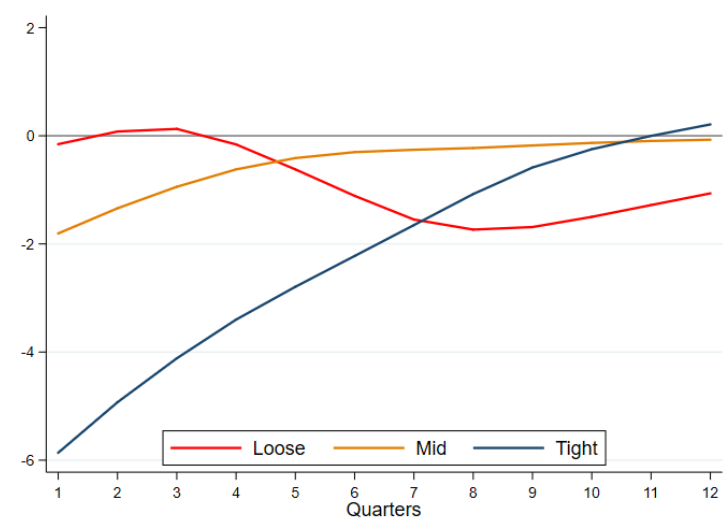

d. Model 3, three lags, Not Boom

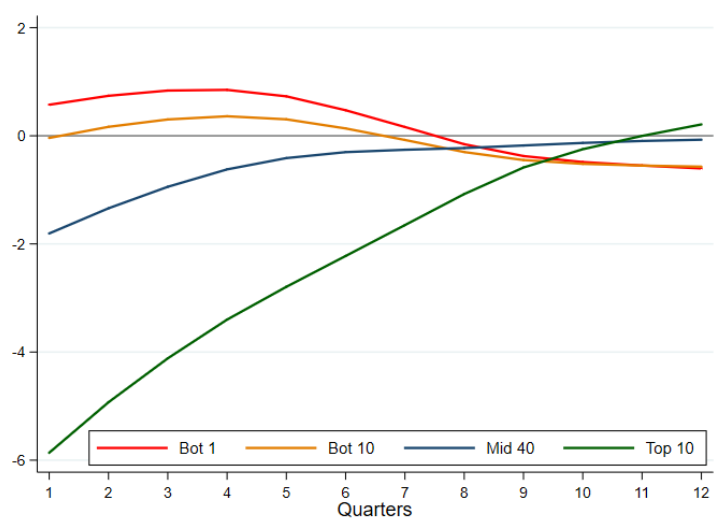

Note: Figures plot the GaR (projected growth at the $5^{\text {th }}$ percentile) at an annual rate for model 3 and for model 3 with lags of independent variables. Real GDP growth, vertical axis, is measured in percent. The GaR projections for each estimation are grouped on initial loosest decile, tightest decile, and mid (middle four deciles) of $\mathrm{FCl}$. Estimates are based on quantile regressions with local projection estimation methods. Advanced economies include 11 countries with data for most from 1973 to 2017. 